\title{
Pinning, de-pinning and re-pinning of a slowly varying rivulet
}

\author{
C. Paterson, ${ }^{*}$ S. K. Wilson ${ }^{\dagger}$ and B. R. Duffy ${ }^{\ddagger}$ \\ Department of Mathematics and Statistics, University of Strathclyde, \\ Livingstone Tower, 26 Richmond Street, Glasgow, G1 1XH, United Kingdom
}

8th February 2013, revised 26th February and 27th March 2013

\begin{abstract}
The solutions for the unidirectional flow of a thin rivulet with prescribed volume flux down an inclined planar substrate are used to describe the locally unidirectional flow of a rivulet with constant width (i.e. pinned contact lines) but slowly varying contact angle as well as the possible pinning and subsequent de-pinning of a rivulet with constant contact angle and the possible depinning and subsequent re-pinning of a rivulet with constant width as they flow in the azimuthal direction from the top to the bottom of a large horizontal cylinder. Despite being the same locally, the global behaviour of a rivulet with constant width can be very different from that of a rivulet with constant contact angle. In particular, while a rivulet with constant non-zero contact angle can always run from the top to the bottom of the cylinder, the behaviour of a rivulet with constant width depends on the value of the width. Specifically, while a narrow rivulet can run all the way from the top to the bottom of the cylinder, a wide rivulet can run from the top of the cylinder only to a critical azimuthal angle. The scenario in which the hitherto pinned contact lines of the rivulet de-pin at the critical azimuthal angle and the rivulet runs from the critical azimuthal angle to the bottom of the cylinder with zero contact angle but slowly varying width is discussed. The pinning and de-pinning of a rivulet with constant contact angle, and the corresponding situation involving the de-pinning and re-pinning of a rivulet with constant width at a non-zero contact angle which generalises the de-pinning at zero contact angle discussed earlier, are described. In the latter situation, the mass of fluid on the cylinder is found to be a monotonically increasing function of the constant width.
\end{abstract}

Keywords: rivulet flow, thin-film flow, contact lines, pinning, de-pinning, re-pinning

\footnotetext{
*Email: colin.paterson@strath.ac.uk

${ }^{\dagger}$ Author for correspondence. Presently also a Visiting Fellow in the Oxford Centre for Collaborative Applied Mathematics (OCCAM), University of Oxford, Mathematical Institute, 24-29 St. Giles', Oxford OX1 3LB. Email: s.k.wilson@strath.ac.uk

${ }^{\ddagger}$ Email: b.r.duffy@strath.ac.uk
} 


\section{Introduction}

The gravity-driven draining of a rivulet of fluid down an inclined substrate is a fundamental fluid mechanics problem of enduring interest, not least because of the wide range of industrial devices and processes to which it is relevant, including heat exchangers (see, for example, Vlasogiannis et al. [1]), trickle-bed reactors (see, for example, Maiti, Khanna and Nigam [2]), various coating processes (see, for example, Kistler and Schweizer [3]), and even the cleaning of the long and narrow tubes found in endoscopes (see, for example, Labib et al. [4]). In particular, the pioneering studies by Towell and Rothfeld [5], Allen and Biggin [6], Bentwich et al. [7], and Davis and co-workers [8]-[10] have led to a substantial body of subsequent work on unidirectional (i.e. rectilinear) rivulet flow. For example, Schmuki and Laso [11] considered the stability of rivulet flow, Kuibin [12], Alekseenko, Geshev and Kuibin [13] and Alekseenko, Bobylev and Markovich [14] considered rivulet flow on the underside of an inclined cylinder, Perazzo and Gratton [15] and Tanasijczuk, Perazzo and Gratton [16] studied sessile and pendent rivulet flow, Myers, Liang and Wetton [17] and Wilson and Duffy [18] considered rivulet flow subject to a constant longitudinal shear stress, and Benilov [19] considered rivulet flow down an inclined substrate and found that sessile and sufficiently narrow pendent rivulets are always stable but that sufficiently wide pendent rivulets are stable only when the incline is sufficiently steep. Duffy and Moffatt [20] used the solution for the unidirectional flow of a thin rivulet with non-zero contact angle and prescribed volume flux to describe the locally unidirectional flow of a rivulet with constant non-zero contact angle but slowly varying width down a slowly varying substrate. In particular, they studied rivulet flow in the azimuthal direction from the top to the bottom of a large horizontal cylinder, and showed that the rivulet becomes wide and flat near the top of the cylinder, but narrow and deep near the bottom of the cylinder. Subsequently Duffy and Wilson [21] performed the corresponding analysis for a rivulet with zero contact angle and, in particular, showed that such rivulets can occur only on the lower half of the cylinder. Various other physical effects, including locally non-planar substrates, thermocapillary effects, viscoplasticity effects, thermoviscosity effects, and a constant longitudinal shear stress, have also been considered (see [21]-[27]).

In the present work we take a rather different approach from the earlier studies and show how the solutions for the unidirectional flow of a thin rivulet with prescribed volume flux down an inclined planar substrate can be used to describe the locally unidirectional flow of a rivulet with constant width but slowly varying contact angle (i.e. pinned contact lines) as well as the possible pinning and subsequent de-pinning of a rivulet with constant contact angle and the possible de-pinning and subsequent re-pinning of a rivulet with constant width as they flow in the azimuthal direction from 
the top to the bottom of a large horizontal cylinder. In particular, we find that, despite being the same locally, the global behaviour of a rivulet with constant width can be very different from that of a rivulet with constant contact angle described by Duffy and Moffatt [20] and Duffy and Wilson $[21]$.

One specific situation in which flow of the type considered in the present work can occur is in the falling-film horizontal-tube evaporators used in a variety of industrial processes, including refrigeration, desalination and petroleum refining. The review article on falling-film evaporation by Ribatski and Jacobi [28] describes how partial film dry-out may occur as a result of a non-uniform distribution of the fluid on the tubes within an evaporator. This non-uniformity can be caused by the gas flow within the evaporator or by uneven draining of the fluid from one tube onto the tube below it in a bundle of horizontal tubes. Typically this draining occurs in one of three main flow regimes, namely a continuous sheet of fluid, an array of separate columns of fluid, or individual drops that drip intermittently. Mitrovic [29] describes various flow regimes and compares the various experimentally determined correlations for the boundaries of the regions in which the different flow regimes occur in Reynolds number - Kapitza number parameter space. In particular, as Mitrovic [29] shows in his Figure 2(h), in the columnar flow regime the fluid in each column can drain around the tubes in an array of separate rivulets or rings of fluid. The flow of both a two-dimensional sheet of fluid and a single three-dimensional column of fluid, falling onto the top of, and draining round to the bottom of, a horizontal cylinder was studied numerically by Hunt [30, 31].

As well as evidently being of direct relevance to falling-film evaporators, the results obtained in the present work may also be relevant to a variety of other practical contexts, such as the rings of fluid on the outer surface of a uniformly rotating horizontal cylinder observed by Moffatt [32] and recently analysed by Leslie, Wilson and Duffy [33], and the banded films of condensed ammonia-water mixtures on the outer surface of a stationary horizontal cylinder observed by Deans and Kucuka [34].

\section{Unidirectional Flow of a Thin Rivulet}

Consider the steady unidirectional flow of a thin symmetric rivulet with semi-width $a$ and volume flux $Q(>0)$ down a planar substrate inclined at an angle $\alpha(0 \leq \alpha \leq \pi)$ to the horizontal. We assume that the fluid is Newtonian with constant viscosity $\mu$, density $\rho$ and coefficient of surface tension $\gamma$, and choose Cartesian coordinates $O x y z$ with the $x$ axis down the line of greatest slope, the $y$ axis horizontal, and the $z$ axis normal to the substrate $z=0$. The velocity $\mathbf{u}=u(y, z) \mathbf{i}$ and the pressure (relative to its ambient value) $p=p(y, z)$ satisfy the familiar mass-conservation and Navier-Stokes 
equations subject to the usual normal and tangential stress balances and the kinematic condition at the free surface $z=h(y)$, the no-slip condition at the substrate $z=0$, and the condition of zero thickness at the contact lines (i.e. $h( \pm a)=0)$. The contact angle is denoted by $\beta=\mp h^{\prime}( \pm a)(\geq 0)$, where the dash denotes differentiation with respect to argument, and the maximum thickness of the rivulet, which always occurs at $y=0$, is denoted by $h_{\mathrm{m}}=h(0)$. We non-dimensionalise $y$ and $a$ with $\ell, z, h$ and $h_{\mathrm{m}}$ with $\delta \ell, u$ with $U=\delta^{2} \rho g \ell^{2} / \mu, Q$ with $\delta \ell^{2} U=\delta^{3} \rho g \ell^{4} / \mu$, and $p$ with $\delta \rho g \ell$, where $g$ is the magnitude of gravitational acceleration, $\ell=(\gamma / \rho g)^{1 / 2}$ is the capillary length, and $\delta$ is the transverse aspect ratio. There is some freedom regarding the definition of $\delta$. When $\beta>0$ we could define $\delta$ using the value of the contact angle by choosing $\delta=\beta$, corresponding to taking $\beta=1$ without loss of generality. Alternatively, we could define $\delta$ using the prescribed value of the flux, denoted by $\bar{Q}(>0)$, by choosing $\delta=\left(\mu \bar{Q} / \rho g \ell^{4}\right)^{1 / 3}$, corresponding to taking $\bar{Q}=1$ without loss of generality. However, for the moment we leave $\delta$ unspecified and retain both $\beta$ and $\bar{Q}$ in order to keep the subsequent presentation as general as possible.

In the general case of non-zero contact angle $\beta>0$ Duffy and Moffatt [20] showed that at leading order in the limit of small transverse aspect ratio $\delta \rightarrow 0$ (i.e. for a thin rivulet) the governing equations are readily solved to yield the velocity $u=\sin \alpha(2 h-z) z / 2$, the pressure $p=\cos \alpha(h-z)-h^{\prime \prime}$, the free surface shape

$$
h(y)=\beta \times \begin{cases}\frac{\cosh m a-\cosh m y}{m \sinh m a} & \text { for } \quad 0 \leq \alpha<\frac{\pi}{2}, \\ \frac{a^{2}-y^{2}}{2 a} & \text { for } \quad \alpha=\frac{\pi}{2}, \\ \frac{\cos m y-\cos m a}{m \sin m a} & \text { for } \quad \frac{\pi}{2}<\alpha \leq \pi,\end{cases}
$$

the maximum thickness of the rivulet

$$
h_{\mathrm{m}}=\beta \times \begin{cases}\frac{1}{m} \tanh \left(\frac{m a}{2}\right) & \text { for } \quad 0 \leq \alpha<\frac{\pi}{2}, \\ \frac{a}{2} & \text { for } \quad \alpha=\frac{\pi}{2} \\ \frac{1}{m} \tan \left(\frac{m a}{2}\right) & \text { for } \quad \frac{\pi}{2}<\alpha \leq \pi,\end{cases}
$$

and the volume flux

$$
Q=\frac{\beta^{3} \sin \alpha}{9 m^{4}} f(m a)
$$

where $m=|\cos \alpha|^{1 / 2}$. The function $f=f(m a)$ appearing in (3) is given by

$$
f(m a)= \begin{cases}15 m a \operatorname{coth}^{3} m a-15 \operatorname{coth}^{2} m a-9 m a \operatorname{coth} m a+4 & \text { for } 0 \leq \alpha<\frac{\pi}{2}, \\ \frac{12}{35}(m a)^{4} & \text { for } \quad \alpha=\frac{\pi}{2}, \\ -15 m a \cot ^{3} m a+15 \cot ^{2} m a-9 m a \cot m a+4 & \text { for } \frac{\pi}{2}<\alpha \leq \pi\end{cases}
$$



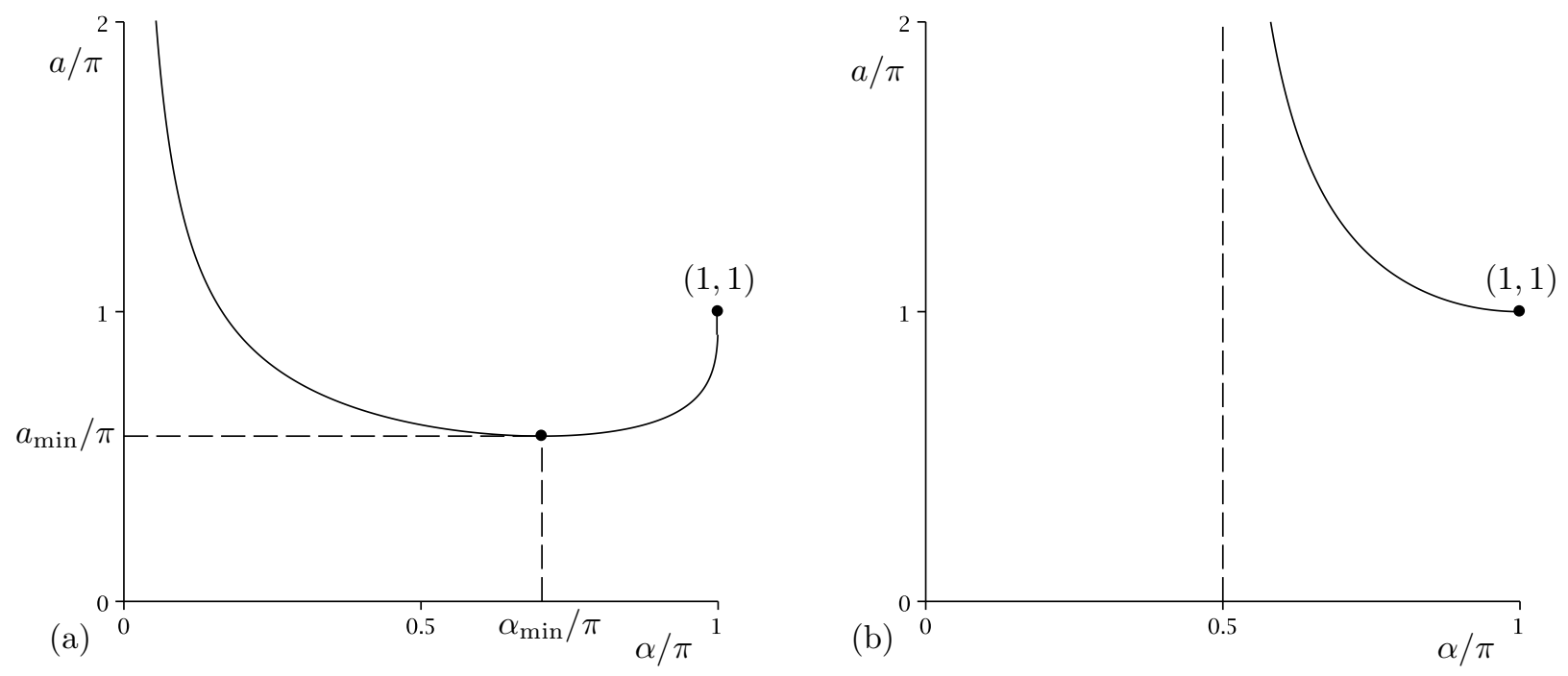

Figure 1: Sketches of the scaled semi-width $a / \pi$ as a function of the scaled azimuthal angle $\alpha / \pi$ for a rivulet with (a) constant non-zero contact angle $\beta=\bar{\beta}>0$ and (b) constant zero contact angle $\beta=\bar{\beta}=0$.

and satisfies $f \sim 12(m a)^{4} / 35 \rightarrow 0$ as $m a \rightarrow 0, f \sim 6 m a-11 \rightarrow \infty$ as $m a \rightarrow \infty$ for $0 \leq \alpha<\pi / 2$, and $f \sim 15 \pi(\pi-m a)^{-3} \rightarrow \infty$ as $m a \rightarrow \pi$ for $\pi / 2<\alpha \leq \pi$.

In the special case of zero contact angle $\beta=0$ we recover the solution for a perfectly wetting fluid described by Duffy and Wilson [21], namely that there is no solution for $0 \leq \alpha \leq \pi / 2$, but

$$
a=\frac{\pi}{m}, \quad h=\frac{h_{\mathrm{m}}}{2}(1+\cos m y), \quad Q=\frac{5 \pi \sin \alpha h_{\mathrm{m}}^{3}}{24 m} \quad \text { for } \quad \frac{\pi}{2}<\alpha \leq \pi .
$$

\section{A Rivulet with Constant Contact Angle}

\subsection{The General Case of Non-Zero Contact Angle $\beta=\bar{\beta}>0$}

Duffy and Moffatt [20] used the solution (1)-(4) to describe the locally unidirectional flow with prescribed flux $Q=\bar{Q}$ down a slowly varying substrate, specifically the flow in the azimuthal direction from the top $\alpha=0$ to the bottom $\alpha=\pi$ of a large horizontal cylinder, of a rivulet with constant non-zero contact angle $\beta=\bar{\beta}>0$ but slowly varying semi-width $a$. Note that here and henceforth "slowly varying" means that the longitudinal aspect ratio $\epsilon=\ell / R$, where $R$ is the radius of the cylinder, satisfies $\epsilon \ll \delta$ so that $\epsilon / \delta \rightarrow 0$ in the limit $\epsilon \rightarrow 0$. Imposing the conditions of prescribed

flux, $Q=\bar{Q}$ with $Q$ given by (3), and of constant non-zero contact angle, $\beta=\bar{\beta}>0$, yields a non-linear algebraic equation for the semi-width $a$ which can, in general, be solved only numerically or asymptotically. Figure 1(a) shows a sketch of the scaled semi-width $a / \pi$ as a function of the scaled 

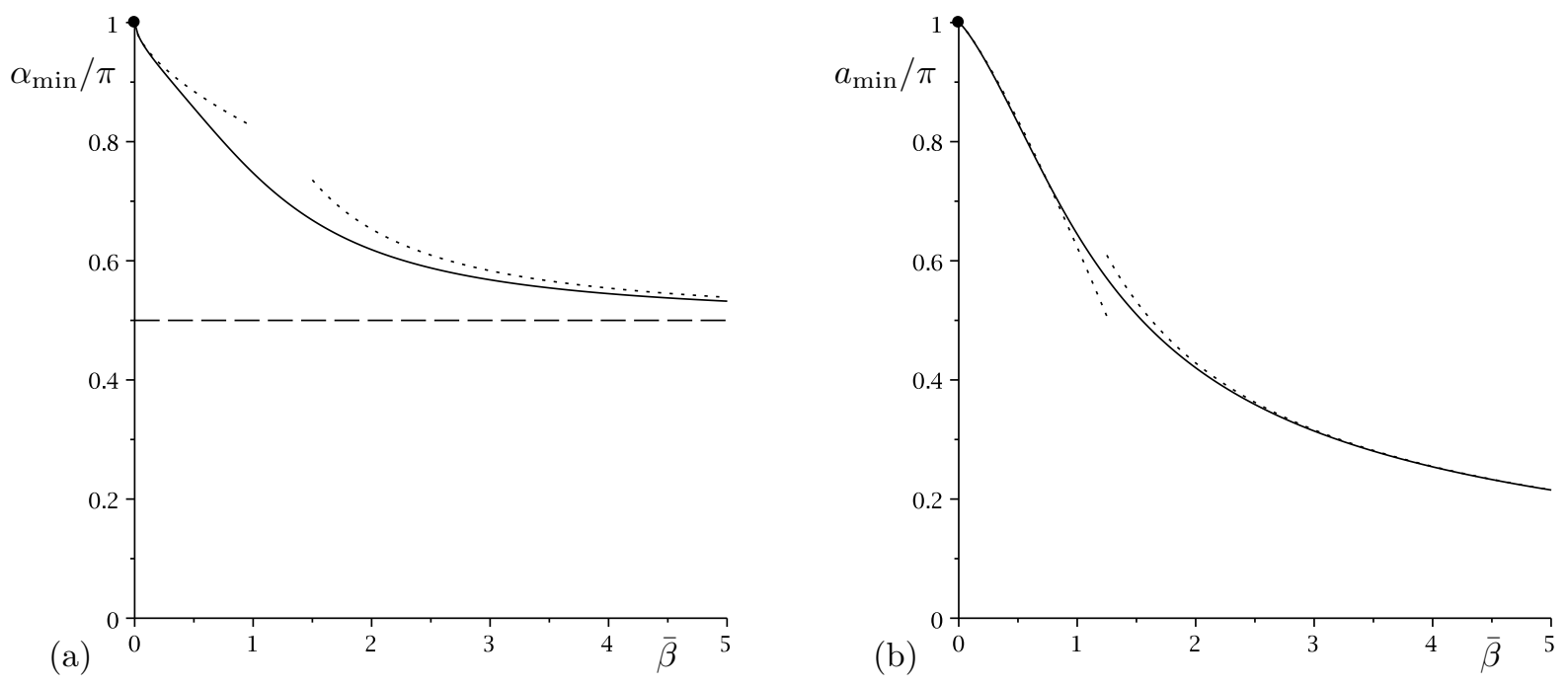

Figure 2: Plots of (a) $\alpha_{\min } / \pi$ and (b) $a_{\min } / \pi$ as functions of the constant contact angle $\bar{\beta}$ when $\bar{Q}=1$, together with their asymptotic behaviour in the limits $\bar{\beta} \rightarrow 0^{+}$given by (6) and $\bar{\beta} \rightarrow \infty$ given by (7), shown with dotted lines.

azimuthal angle $\alpha / \pi$ when $\beta=\bar{\beta}>0$. For all values of $\bar{Q}$ there is a slowly varying rivulet that runs all the way from $\alpha=0$ [where $a=O\left(\alpha^{-1}\right) \rightarrow \infty$ and $h_{\mathrm{m}} \rightarrow 1^{+}$as $\alpha \rightarrow 0^{+}$] to $\alpha=\pi$ [where $a \rightarrow \pi^{-}$ and $h_{\mathrm{m}}=O(\pi-\alpha)^{-1 / 3} \rightarrow \infty$ as $\alpha \rightarrow \pi^{-}$. The rivulet does not have top-to-bottom symmetry; its semi-width $a$ has a single minimum, denoted by $a=a_{\min }(<\pi)$ and occurring at $\alpha=\alpha_{\min }$, on the lower half of the cylinder (i.e. for $\pi / 2<\alpha \leq \pi$ ), and its maximum thickness $h_{\mathrm{m}}$ either increases monotonically or has a single maximum and a single minimum on the upper half of the cylinder (i.e. for $0 \leq \alpha<\pi / 2)$. Furthermore, in the limit of small flux, $\bar{Q} \rightarrow 0^{+}$, the rivulet satisfies $a=O\left(\bar{Q}^{1 / 4}\right)$ and $h_{\mathrm{m}}=O\left(\bar{Q}^{1 / 4}\right)$ while in the limit of large flux, $\bar{Q} \rightarrow \infty$, it satisfies $a=O(\bar{Q})$ and $h_{\mathrm{m}}=O(1)$ on the upper half of the cylinder, $a=O\left(\bar{Q}^{1 / 4}\right)$ and $h_{\mathrm{m}}=O\left(\bar{Q}^{1 / 4}\right)$ at $\alpha=\pi / 2$, and $a=O(1)$ and $h_{\mathrm{m}}=O\left(\bar{Q}^{1 / 3}\right)$ on the lower half of the cylinder. Since the location and value of the minimum semi-width are important in what follows, Figure 2 shows plots of $\alpha_{\min } / \pi$ and $a_{\min } / \pi$ as functions of the constant contact angle $\bar{\beta}$, and shows that both are monotonically decreasing functions of $\bar{\beta}$ satisfying

$$
\alpha_{\min } \sim \pi-\left(\frac{40 \bar{\beta}^{3}}{81 \pi^{2} \bar{Q}}\right)^{\frac{1}{5}} \rightarrow \pi^{-} \quad \text { and } \quad a_{\min } \sim \pi-\frac{5 \pi}{4}\left(\frac{40 \bar{\beta}^{3}}{81 \pi^{2} \bar{Q}}\right)^{\frac{2}{5}} \rightarrow \pi^{-}
$$

as $\bar{\beta} \rightarrow 0^{+}$, and

$$
\alpha_{\min } \sim \frac{\pi}{2}+\frac{2}{9}\left(\frac{105 \bar{Q}}{4 \bar{\beta}^{3}}\right)^{\frac{1}{2}} \rightarrow \frac{\pi}{2}^{+} \quad \text { and } \quad a_{\min } \sim\left(\frac{105 \bar{Q}}{4 \bar{\beta}^{3}}\right)^{\frac{1}{4}} \rightarrow 0^{+}
$$

as $\bar{\beta} \rightarrow \infty$. 


\subsection{The Special Case of Zero Contact Angle $\beta=\bar{\beta}=0$}

Duffy and Wilson [21] used the solution (5) to describe the corresponding flow of a rivulet with zero contact angle $\beta=\bar{\beta}=0$ (i.e. a perfectly wetting fluid). Specifically, imposing the condition of prescribed flux, $Q=\bar{Q}$ with $Q$ given by (5), yields an explicit solution for the maximum thickness $h_{\mathrm{m}}=h_{\mathrm{m} 0}$, where

$$
h_{\mathrm{m} 0}=\left(\frac{24 \bar{Q} m}{5 \pi \sin \alpha}\right)^{\frac{1}{3}}=\left(\frac{24 \bar{Q}|\cos \alpha|^{1 / 2}}{5 \pi \sin \alpha}\right)^{\frac{1}{3}} .
$$

Figure 1(b) shows a sketch of the scaled semi-width $a / \pi$ as a function of the scaled azimuthal angle $\alpha / \pi$ when $\beta=\bar{\beta}=0$. For all values of $\bar{Q}$ there is a slowly varying rivulet on the lower half of the cylinder with monotonically decreasing semi-width $a=\pi / m$ and monotonically increasing maximum thickness $h_{\mathrm{m}}=h_{\mathrm{m} 0}$ that runs from $\alpha=\pi / 2^{+}$[where $a=O(\alpha-\pi / 2)^{-1 / 2} \rightarrow \infty$ and $h_{\mathrm{m}}=O(\alpha-\pi / 2)^{1 / 6} \rightarrow 0^{+}$as $\alpha \rightarrow \pi / 2^{+}$] to $\alpha=\pi$ [where $a \rightarrow \pi^{+}$and $h_{\mathrm{m}}=O(\pi-\alpha)^{-1 / 3} \rightarrow \infty$ as $\left.\alpha \rightarrow \pi^{-}\right]$. Note that, unlike in the general case of non-zero contact angle $\bar{\beta}>0$, in which there is an infinite mass of fluid on the cylinder, in the special case of zero contact angle $\beta=\bar{\beta}=0$ the mass of fluid on the cylinder, denoted by $M$ and non-dimensionalised with $\delta \rho \ell^{2} R$, is given by

$$
M=\int_{\frac{\pi}{2}}^{\pi} \int_{-a}^{+a} h \mathrm{~d} y \mathrm{~d} \alpha=\int_{\frac{\pi}{2}}^{\pi} \frac{\pi h_{\mathrm{m}}}{m} \mathrm{~d} \alpha=\left(\frac{6 \pi^{2} \bar{Q}}{5}\right)^{\frac{1}{3}} C,
$$

where the constant $C$ is given by

$$
C=\int_{0}^{\pi} \frac{\mathrm{d} \alpha}{(\sin \alpha)^{\frac{1}{3}}}=\frac{\sqrt{\pi} \Gamma\left(\frac{1}{3}\right)}{\Gamma\left(\frac{5}{6}\right)} \simeq 4.2065 .
$$

\section{A Rivulet with Constant Width}

The solutions (1)-(4) and (5) can also be used to describe the locally unidirectional flow with prescribed flux $Q=\bar{Q}$ from the top $\alpha=0$ to the bottom $\alpha=\pi$ of a large horizontal cylinder of a rivulet with constant semi-width $a=\bar{a}(>0)$ (i.e. pinned contact lines) but slowly varying contact angle $\beta(\geq 0)$. Imposing the conditions of prescribed flux, $Q=\bar{Q}$ with $Q$ given by (3), and of constant semi-width, $a=\bar{a}$, yields an explicit solution for the contact angle $\beta$, namely

$$
\beta=\left(\frac{9 \bar{Q} m^{4}}{f(m \bar{a}) \sin \alpha}\right)^{\frac{1}{3}}=\left(\frac{9 \bar{Q} \cos ^{2} \alpha}{f\left(|\cos \alpha|^{1 / 2} \bar{a}\right) \sin \alpha}\right)^{\frac{1}{3}}
$$

The solution (11) reveals that, unlike in the case of constant contact angle described in section 3 (in which the dependence of $a$ on $\bar{Q}$ is not straightforward), in this case $\beta$ is simply proportional to $\bar{Q}^{1 / 3}$ for all values of $\alpha$ and $\bar{a}$. Moreover, as in the case of constant contact angle, the rivulet does not 

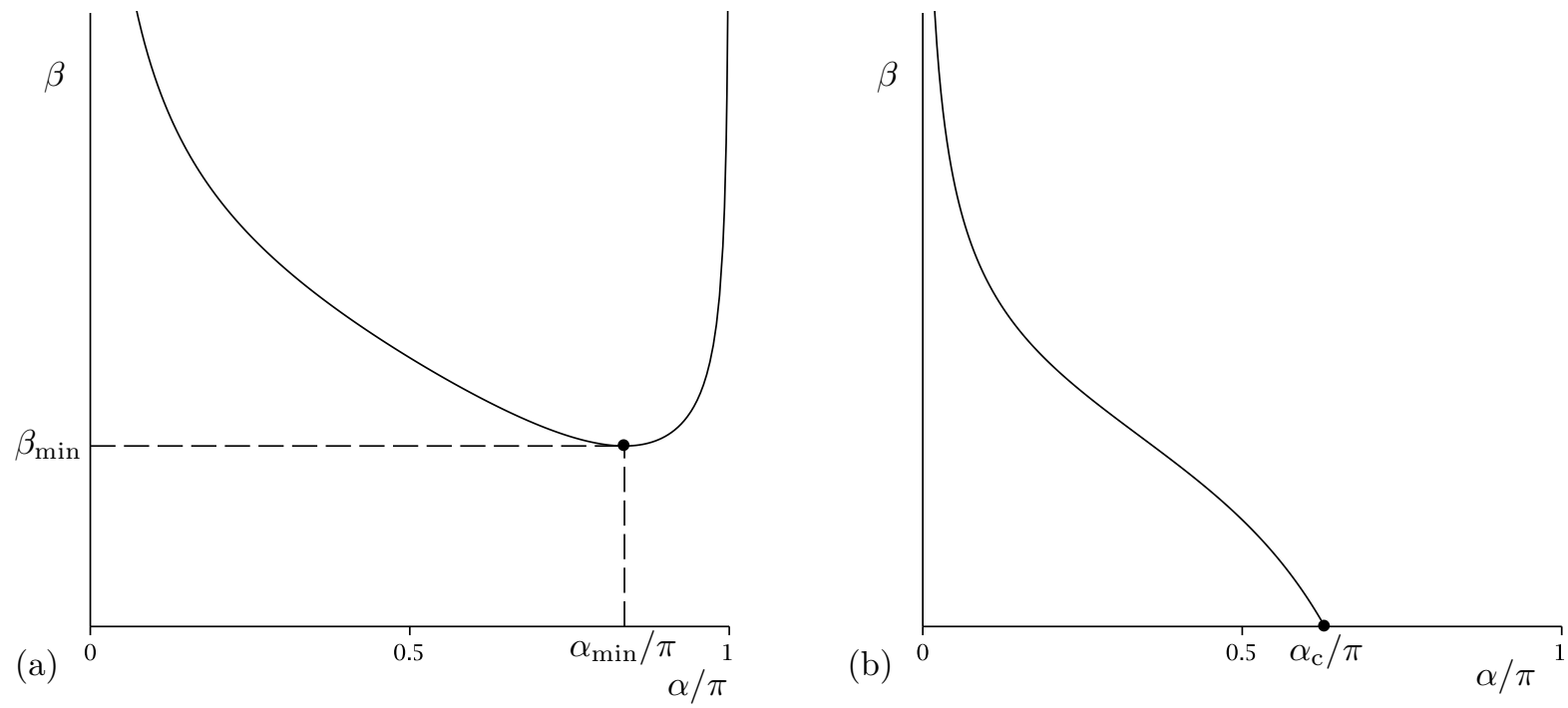

Figure 3: Sketches of the contact angle $\beta$ as a function of the scaled azimuthal angle $\alpha / \pi$ for (a) a "narrow" rivulet with constant semi-width $a=\bar{a}<\pi$ and (b) a "wide" rivulet with constant semi-width $a=\bar{a}>\pi$. For brevity, the marginal case $a=\bar{a}=\pi$ is not shown.

have top-to-bottom symmetry. Inspection of the solution (11) also reveals that, unlike in the case of constant contact angle (in which the behaviour is qualitatively the same for all values of the contact angle), the behaviour of the rivulet is qualitatively different for a "narrow" rivulet with $a=\bar{a}<\pi$, in the marginal case $a=\bar{a}=\pi$, and for a "wide" rivulet with $a=\bar{a}>\pi$. We shall therefore describe the behaviour of the rivulet in each of these three cases separately in the next three subsections.

\subsection{A Narrow Rivulet with $a=\bar{a}<\pi$}

Figure 3(a) shows a sketch of the contact angle $\beta$ as a function of the scaled azimuthal angle $\alpha / \pi$ for a narrow rivulet with constant semi-width $a=\bar{a}<\pi$. When $\bar{a}<\pi$ for all values of $\bar{Q}$ there is a slowly varying rivulet that runs all the way from $\alpha=0$ to $\alpha=\pi$, and its contact angle $\beta$ has a single minimum, denoted by $\beta=\beta_{\min }$ and occurring at $\alpha=\alpha_{\min }$, on the lower half of the cylinder (i.e. for $\pi / 2<\alpha<\pi)^{1}$, and its maximum thickness $h_{\mathrm{m}}$ has a single minimum on the upper half of the cylinder (i.e. for $0<\alpha<\pi / 2$ ). Since the location and value of the minimum contact angle are important in what follows, Figure 4 shows plots of $\alpha_{\min } / \pi$ and $\beta_{\min }$ as functions of the scaled constant semi-width $\bar{a} / \pi$, and shows that $\alpha_{\min }$ is a monotonically increasing function of $\bar{a}$ and $\beta_{\min }$

\footnotetext{
${ }^{1}$ Note that $\alpha_{\min }$ is independent of $\bar{Q}$.
} 

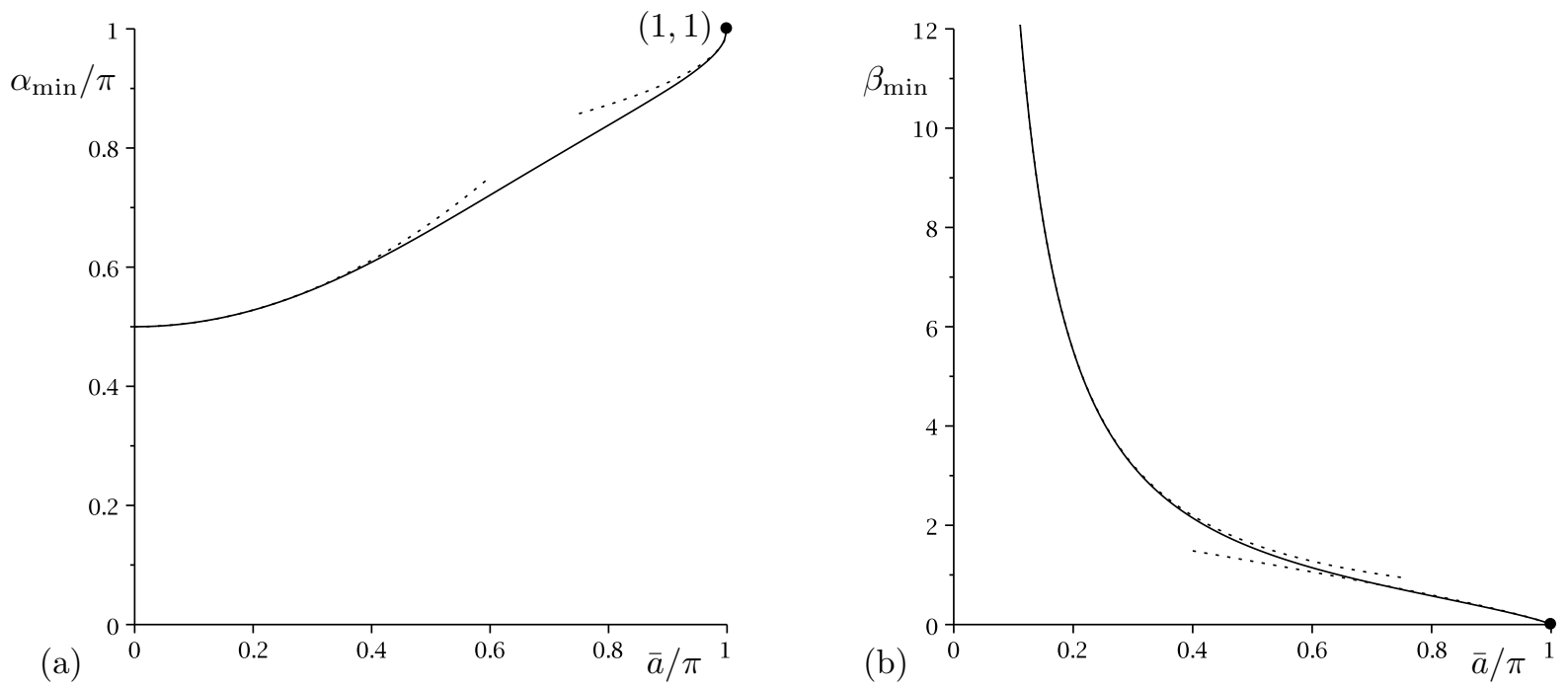

Figure 4: Plots of (a) $\alpha_{\min } / \pi$ and (b) $\beta_{\min }$ as functions of the scaled constant semi-width $\bar{a} / \pi$ when $\bar{Q}=1$, together with their asymptotic behaviour in the limits $\bar{a} \rightarrow 0^{+}$given by (12) and $\bar{a} \rightarrow \infty$ given by (13), shown with dotted lines.

is a monotonically decreasing function of $\bar{a}$ satisfying

$$
\alpha_{\min } \sim \frac{\pi}{2}+\frac{2 \bar{a}^{2}}{9} \rightarrow \frac{\pi}{2} \quad \text { and } \quad \beta_{\min } \sim\left(\frac{105 \bar{Q}}{4 \bar{a}^{4}}\right)^{\frac{1}{3}} \rightarrow \infty
$$

as $\bar{a} \rightarrow 0^{+}$, and

$$
\alpha_{\min } \sim \pi-\left(\frac{4(\pi-\bar{a})}{5 \pi}\right)^{\frac{1}{2}} \rightarrow \pi^{-} \quad \text { and } \quad \beta_{\min } \sim \frac{6}{5}\left(\frac{9 \bar{Q}^{2}}{20 \pi}\right)^{\frac{1}{6}}(\pi-\bar{a})^{\frac{5}{6}} \rightarrow 0^{+}
$$

as $\bar{a} \rightarrow \pi^{-}$. The rivulet becomes deep near the top and the bottom of the cylinder according to

$$
\beta \sim\left(\frac{9 \bar{Q}}{f(\bar{a}) \alpha}\right)^{\frac{1}{3}} \rightarrow \infty \quad \text { and } \quad h_{\mathrm{m}} \sim\left(\frac{9 \bar{Q}}{f(\bar{a}) \alpha}\right)^{\frac{1}{3}} \tanh \left(\frac{\bar{a}}{2}\right) \rightarrow \infty
$$

as $\alpha \rightarrow 0^{+}$, and

$$
\beta \sim\left(\frac{9 \bar{Q}}{f(\bar{a})(\pi-\alpha)}\right)^{\frac{1}{3}} \rightarrow \infty \quad \text { and } \quad h_{\mathrm{m}} \sim\left(\frac{9 \bar{Q}}{f(\bar{a})(\pi-\alpha)}\right)^{\frac{1}{3}} \tan \left(\frac{\bar{a}}{2}\right) \rightarrow \infty
$$

as $\alpha \rightarrow \pi^{-}$(so that the thin-film approximation ultimately fails in these limits); also $\beta$ and $h_{\mathrm{m}}$ take the $O(1)$ values

$$
\beta=\left(\frac{105 \bar{Q}}{4 \bar{a}^{4}}\right)^{\frac{1}{3}} \text { and } \quad h_{\mathrm{m}}=\left(\frac{105 \bar{Q}}{32 \bar{a}}\right)^{\frac{1}{3}}
$$

at $\alpha=\pi / 2$. In the limit of a narrow rivulet, $\bar{a} \rightarrow 0^{+}$, the rivulet becomes narrow and deep everywhere according to

$$
\beta \sim\left(\frac{105 \bar{Q}}{4 \bar{a}^{4} \sin \alpha}\right)^{\frac{1}{3}} \rightarrow \infty \quad \text { and } \quad h_{\mathrm{m}} \sim\left(\frac{105 \bar{Q}}{32 \bar{a} \sin \alpha}\right)^{\frac{1}{3}} \rightarrow \infty
$$




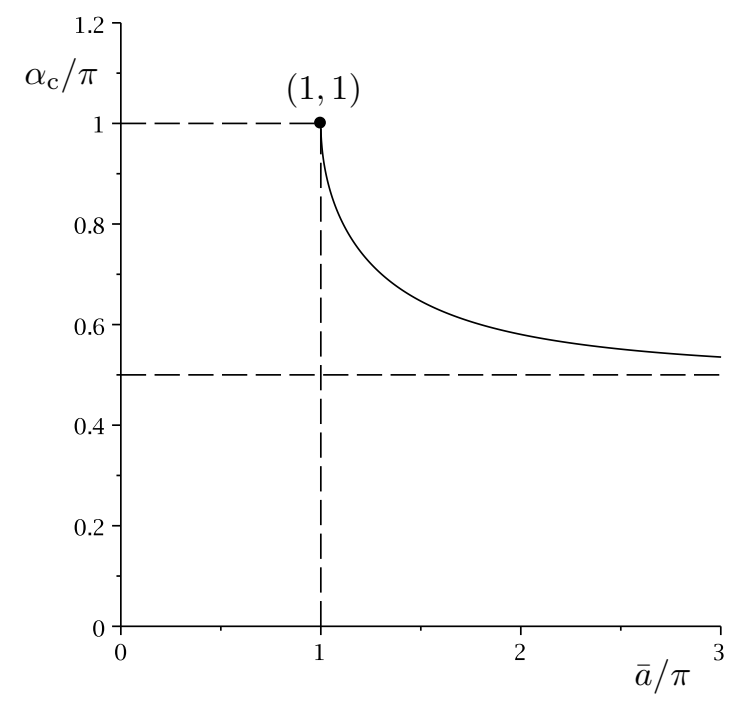

Figure 5: Plot of the scaled critical azimuthal angle $\alpha_{\mathrm{c}} / \pi$ as a function of the scaled constant semiwidth $\bar{a} / \pi$.

\subsection{The Marginal Case $a=\bar{a}=\pi$}

In the marginal case $a=\bar{a}=\pi$ (not shown in Figure 3 for brevity) the rivulet behaves qualitatively the same as in the case of a narrow rivulet with $a=\bar{a}<\pi$ described in subsection 4.1, except that, since in this case $\beta=0$ at $\alpha=\pi$, instead of satisfying (15) the rivulet becomes deep with zero contact angle and finite semi-width $\pi$ near the bottom of the cylinder according to

$$
\beta \sim\left(\frac{3 \pi^{2} \bar{Q}(\pi-\alpha)^{5}}{320}\right)^{\frac{1}{3}} \rightarrow 0^{+} \quad \text { and } \quad h_{\mathrm{m}} \sim\left(\frac{24 \bar{Q}}{5 \pi(\pi-\alpha)}\right)^{\frac{1}{3}} \rightarrow \infty
$$

as $\alpha \rightarrow \pi^{-}$.

\subsection{A Wide Rivulet with $a=\bar{a}>\pi$}

Figure 3(b) shows a sketch of the contact angle $\beta$ as a function of the scaled azimuthal angle $\alpha / \pi$ for a wide rivulet with constant semi-width $a=\bar{a}>\pi$. Unlike when $\bar{a} \leq \pi$, when $\bar{a}>\pi$ for all values of $\bar{Q}$ there is a slowly varying rivulet that runs from $\alpha=0$ only as far as a critical azimuthal angle $\alpha=\alpha_{\mathrm{c}}$ on the lower half of the cylinder (i.e. for $\left.\pi / 2<\alpha<\pi\right)^{2}$, and its contact angle $\beta$ is a monotonically decreasing function of $\alpha$, attaining its minimum physically realisable value of zero at $\alpha=\alpha_{\mathrm{c}}$, where the critical azimuthal angle $\alpha_{\mathrm{c}}$ is given by solving $m \bar{a}=\pi$ to obtain

$$
\alpha_{\mathrm{c}}=\cos ^{-1}\left(-\frac{\pi^{2}}{\bar{a}^{2}}\right) \quad \text { for } \quad \bar{a}>\pi
$$

\footnotetext{
${ }^{2}$ Note that $\alpha_{\mathrm{c}}$ is independent of $\bar{Q}$.
} 
and is a monotonically decreasing function of $\bar{a}$ satisfying $\alpha_{\mathrm{c}}=\pi+O(\bar{a}-\pi)^{1 / 2} \rightarrow \pi^{-}$as $\bar{a} \rightarrow \pi^{+}$ and $\alpha_{\mathrm{c}}=\pi / 2+O\left(\bar{a}^{-2}\right) \rightarrow \pi / 2^{+}$as $\bar{a} \rightarrow \infty$. Figure 5 shows the scaled critical azimuthal angle $\alpha_{\mathrm{c}} / \pi$ plotted as a function of the scaled constant semi-width $\bar{a} / \pi$. The rivulet again becomes deep near the top of the cylinder according to (14) and again $\beta$ and $h_{\mathrm{m}}$ take the $O(1)$ values given by (16) at $\alpha=\pi / 2$. At $\alpha=\alpha_{\mathrm{c}}$ the rivulet has zero contact angle $\beta=0$, semi-width $a=\bar{a}$, and maximum thickness $h_{\mathrm{m}}=h_{\mathrm{mc}}$, where

$$
h_{\mathrm{mc}}=\left(\frac{24 \bar{Q}}{5 \bar{a} \sin \alpha_{\mathrm{c}}}\right)^{\frac{1}{3}}=\left(\frac{24 \bar{a} \bar{Q}}{5 \sqrt{\bar{a}^{4}-\pi^{4}}}\right)^{\frac{1}{3}} .
$$

In particular, as $\alpha \rightarrow \alpha_{\mathrm{c}}^{-}$we find that $\beta \rightarrow 0^{+}$according to

$$
\beta=\left(\frac{3\left(\bar{a}^{4}-\pi^{4}\right) \bar{Q}}{40 \bar{a}^{2}}\right)^{\frac{1}{3}}\left(\alpha_{\mathrm{c}}-\alpha\right)+O\left(\alpha_{\mathrm{c}}-\alpha\right)^{2},
$$

$a \equiv \bar{a}$, and $h_{\mathrm{m}} \rightarrow h_{\mathrm{mc}}^{-}$according to

$$
h_{\mathrm{m}}=h_{\mathrm{mc}}+\frac{\left(\bar{a}^{4}+\pi^{4}\right) h_{\mathrm{mc}}}{6 \pi^{2} \sqrt{\bar{a}^{4}-\pi^{4}}}\left(\alpha-\alpha_{\mathrm{c}}\right)+O\left(\alpha-\alpha_{\mathrm{c}}\right)^{2} .
$$

However, beyond $\alpha=\alpha_{\mathrm{c}}$ the solution for $\beta$ given by (11) is no longer physically realisable because it always predicts that $h<0$ somewhere in the interval $y=-\bar{a}$ to $y=+\bar{a}$, and so an alternative description of the behaviour beyond $\alpha=\alpha_{\mathrm{c}}$ is required. Physically it is possible that the rivulet simply falls off the cylinder at $\alpha=\alpha_{\mathrm{c}}$ or that the flow becomes unsteady beyond $\alpha=\alpha_{\mathrm{c}}$. However, an alternative (and possibly more likely) scenario in which steady rivulet flow still occurs is that the hitherto pinned contact lines of the rivulet de-pin at $\alpha=\alpha_{\mathrm{c}}$, and that the rivulet runs from $\alpha=\alpha_{\mathrm{c}}$ to the bottom of the cylinder $\alpha=\pi$ with zero contact angle according to the solution in the case $\beta=0$ given by (5) and (8), with monotonically decreasing semi-width $a=\pi / m(\pi \leq a \leq \bar{a})$ and monotonically increasing maximum thickness $h_{\mathrm{m}}=h_{\mathrm{m} 0}\left(\geq h_{\mathrm{mc}}\right)$. In particular, as $\alpha \rightarrow \alpha_{\mathrm{c}}^{+}$we find that $\beta \equiv 0, a \rightarrow \bar{a}^{-}$according to

$$
a=\bar{a}-\frac{\bar{a} \sqrt{\bar{a}^{4}-\pi^{4}}}{2 \pi^{2}}\left(\alpha-\alpha_{\mathrm{c}}\right)+O\left(\alpha-\alpha_{\mathrm{c}}\right)^{2}
$$

and $h_{\mathrm{m}} \rightarrow h_{\mathrm{mc}}^{+}$according to (22), so that the solutions in $\alpha<\alpha_{\mathrm{c}}$ and $\alpha>\alpha_{\mathrm{c}}$ join continuously (but not smoothly) at $\alpha=\alpha_{\mathrm{c}}$. This latter scenario is a special case of the behaviour which will be discussed in section 6 , in which we consider the de-pinning and re-pinning of a rivulet with constant width at a prescribed (and, in general, non-zero) value of the contact angle $\beta=\bar{\beta}(\geq 0)$. In particular, when the rivulet de-pins at zero contact angle $\beta=\bar{\beta}=0$ it becomes deep with zero contact angle and finite semi-width $\pi$ near the bottom of the cylinder according to

$$
a=\pi+\frac{\pi}{4}(\pi-\alpha)^{2}+O(\pi-\alpha)^{4} \rightarrow \pi^{+} \quad \text { and } \quad h_{\mathrm{m}} \sim\left(\frac{24 \bar{Q}}{5 \pi(\pi-\alpha)}\right)^{\frac{1}{3}} \rightarrow \infty
$$


as $\alpha \rightarrow \pi^{-}$, and in the limit of a wide rivulet on the upper half of the cylinder, $\bar{a} \rightarrow \infty$, (in which $\left.\alpha_{\mathrm{c}} \rightarrow \pi / 2^{+}\right)$the rivulet becomes wide and flat on the upper half of the cylinder according to

$$
\beta \sim\left(\frac{3 \bar{Q} m^{3}}{2 \bar{a} \sin \alpha}\right)^{\frac{1}{3}} \rightarrow 0^{+} \quad \text { and } \quad h_{\mathrm{m}} \sim\left(\frac{3 \bar{Q}}{2 \bar{a} \sin \alpha}\right)^{\frac{1}{3}} \rightarrow 0^{+}
$$

and is given by the solution in the case $\beta=0$ given by (5) and (8) on the lower half of the cylinder.

\subsection{Rivulet Profiles}

The behaviour for both $\bar{a} \leq \pi$ and $\bar{a}>\pi$ is illustrated in Figure 6, which shows plots of the contact

angle $\beta$, the scaled semi-width $a / \pi$, and the maximum thickness $h_{\mathrm{m}}$ as functions of the scaled angle $\alpha / \pi$ for a range of values of $\bar{a} / \pi$ when $\bar{Q}=1$. In particular, Figure 6 shows that de-pinning occurs at $\alpha=\alpha_{\mathrm{c}}$ for $\bar{a} / \pi>1$. Figure 7 shows typical cross-sectional profiles of the rivulet in the cases (a) $\bar{a}=2(<\pi)$ and (b) $\bar{a}=5(>\pi)$, and Figure 8 shows sketches of the rivulet in the same two cases, namely (a) $\bar{a}<\pi$, in which the rivulet is never de-pinned, and (b) $\bar{a}>\pi$, in which the rivulet is de-pinned and has zero contact angle in the interval $\alpha_{\mathrm{c}}<\alpha \leq \pi$.

\section{Pinning and De-Pinning of a Rivulet with Constant Contact An- gle $\beta=\bar{\beta}$ at $a=\bar{a}$}

As we described in section 3, the semi-width $a$ of a slowly varying rivulet with constant non-zero contact angle $\beta=\bar{\beta}>0$ is unbounded at $\alpha=0$ (i.e. the rivulet is infinitely wide at the top of the cylinder), has a single minimum value of $a=a_{\min }(<\pi)$ at $\alpha=\alpha_{\min }$ on the lower half of the cylinder and takes the value $a=\pi$ at $\alpha=\pi$, while in the special case of zero contact angle $\beta=\bar{\beta}=0$ the semi-width is unbounded at $\alpha=\pi / 2$ and decreases to the value $a=\pi$ at $\alpha=\pi$. In practice, however, there could be a minimum physically realisable value of the semi-width, denoted by $a=\bar{a}$, at which the contact lines become pinned. Evidently the behaviour of the rivulet in this situation will be qualitatively different for $\bar{a} \leq \pi$ and $\bar{a}>\pi$.

\section{$5.1 \bar{a} \leq \pi$}

When $\bar{a} \leq a_{\min }(<\pi)$ the semi-width is always greater than or equal to $\bar{a}$ and hence pinning and de-pinning do not occur, and so the rivulet behaves exactly as described in section 3 . However, when $a_{\text {min }}<\bar{a}<\pi$ the rivulet runs from $\alpha=0$ with constant non-zero contact angle $\beta=\bar{\beta}>0$ but decreasing semi-width $a$ as described in section 3 until it reaches the value $a=\bar{a}$ at $\alpha=\alpha_{\text {pin }}$ 

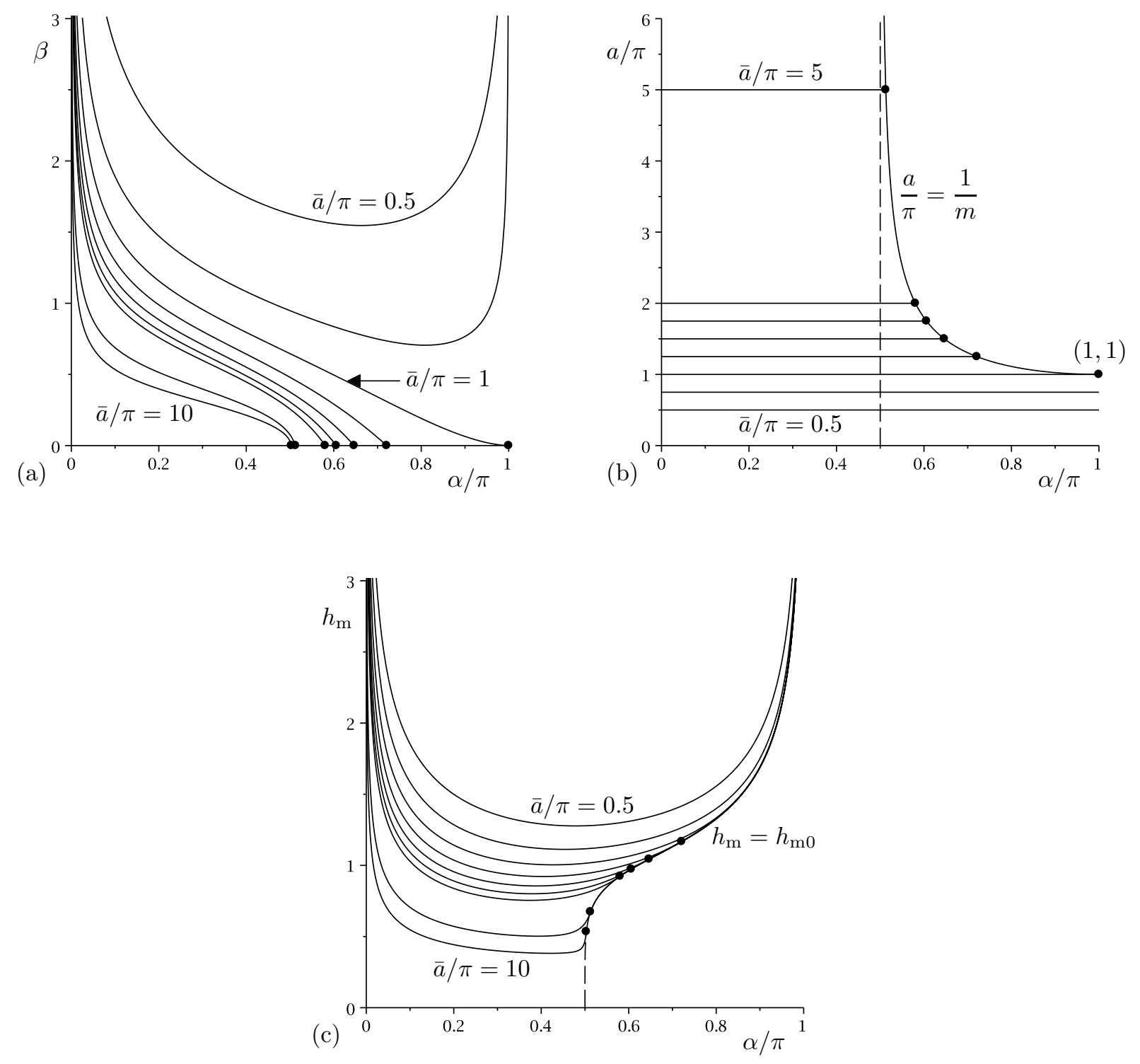

Figure 6: Plots of (a) the contact angle $\beta$, (b) the scaled semi-width $a / \pi$, and (c) the maximum thickness $h_{\mathrm{m}}$ as functions of the scaled angle $\alpha / \pi$ for $\bar{a} / \pi=0.5,0.75,1,1.25,1.5,1.75,2,5,10$ when $\bar{Q}=1$ for a rivulet whose contact lines de-pin at zero contact angle $\beta=\bar{\beta}=0$. The corresponding solutions for a rivulet with zero contact angle $\beta=0$ given by (5) and (8) are shown with dashed lines (visible only in part (c)). De-pinning occurs at $\alpha=\alpha_{\mathrm{c}}$ for $\bar{a} / \pi>1$, and the points at which this happens are denoted by dots. 

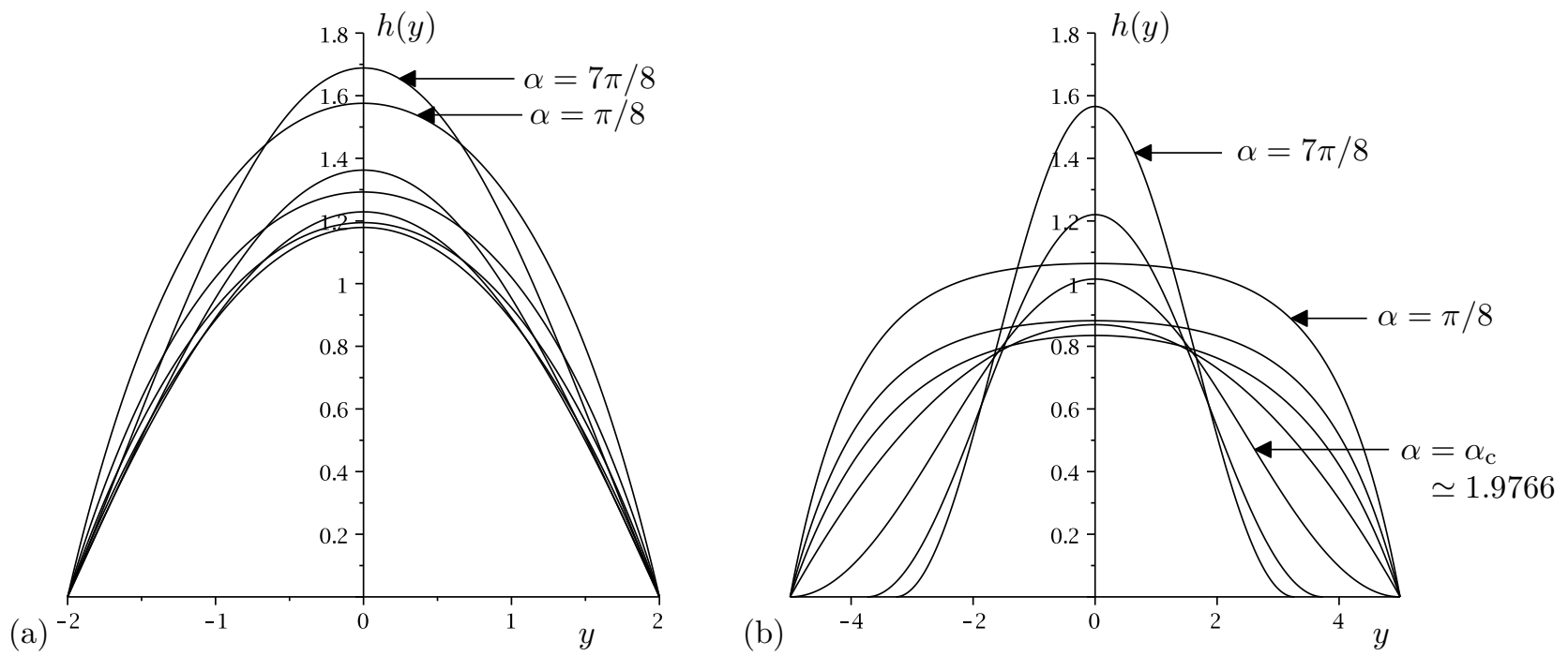

Figure 7: Cross-sectional profiles $h(y)$ when $\bar{Q}=1$ in the cases (a) $\bar{a}=2(<\pi)$ for $\alpha=\pi / 8, \pi / 4$, $3 \pi / 8, \pi / 2,5 \pi / 8,3 \pi / 4,7 \pi / 8$ and (b) $\bar{a}=5(>\pi)$ for $\alpha=\pi / 8, \pi / 4,3 \pi / 8, \pi / 2, \alpha_{\mathrm{c}} \simeq 1.9766,3 \pi / 4$, $7 \pi / 8$. For clarity, the two parts of this figure use the same vertical but different horizontal ranges.

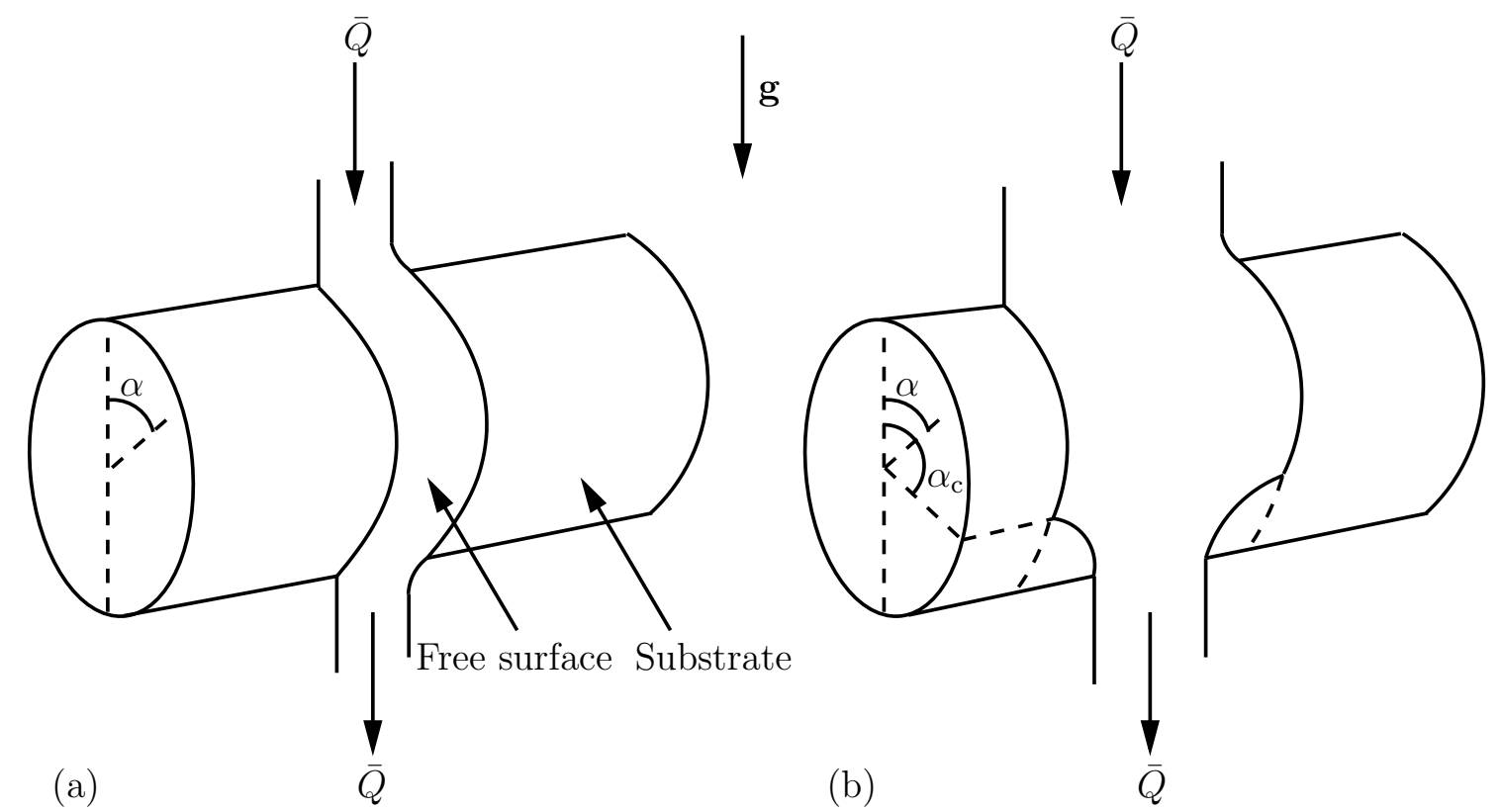

Figure 8: Sketches of a slowly varying rivulet with prescribed flux $\bar{Q}$ with (when not de-pinned with zero contact angle $\beta=0$ and slowly varying semi-width $a$ ) constant semi-width $a=\bar{a}$ and slowly varying contact angle $\beta$ that runs from the top $\alpha=0$ to the bottom $\alpha=\pi$ of a large horizontal cylinder, in the cases (a) $\bar{a}<\pi$, in which the rivulet is never de-pinned, and (b) $\bar{a}>\pi$, in which the rivulet is de-pinned and has zero contact angle in the interval $\alpha_{\mathrm{c}}<\alpha \leq \pi$. 
$\left(0<\alpha_{\text {pin }}<\alpha_{\text {min }}\right)$ at which the contact lines pin. The rivulet then runs from $\alpha=\alpha_{\text {pin }}$ with constant semi-width $a=\bar{a}$ but varying contact angle $\beta$ as described in section 4 until it reaches $\alpha=\alpha_{\text {depin }}$ $\left(\alpha_{\min }<\alpha_{\text {depin }}<\pi\right)$ at which the contact lines de-pin. The rivulet then runs from $\alpha=\alpha_{\text {depin }}$ to $\alpha=\pi$ with constant contact angle $\beta=\bar{\beta}>0$ but increasing semi-width $a$ as described in section 3 . Here $\alpha=\alpha_{\text {pin }}$ and $\alpha=\alpha_{\text {depin }}$ are the appropriate solutions of the equation $Q=\bar{Q}$ with $Q$ given by (3), $a=\bar{a}$ and $\beta=\bar{\beta}$. In the marginal case $\bar{a}=\pi$ we have $\alpha_{\text {depin }}=\pi$ and so de-pinning does not occur. Expressed another way, in the general case of constant non-zero contact angle $\bar{\beta}>0$ pinning and de-pinning occur when $\bar{\beta}>\bar{\beta}_{\mathrm{c}}$, where the value of $\bar{\beta}_{\mathrm{c}}$ corresponds to $a_{\min }=\bar{a}$. In the special case of pinning at zero contact angle $\beta=\bar{\beta}=0$ we have $a=\pi / m \geq \pi \geq \bar{a}$, and so pinning and de-pinning do not occur. In the limit $\bar{\beta} \rightarrow \infty$ we have $\alpha_{\text {pin }} \rightarrow 0^{+}$and $\alpha_{\text {depin }} \rightarrow \pi^{-}$and so recover the solution for a rivulet with constant semi-width $a=\bar{a} \leq \pi$ described in section 4 .

The behaviour when $\bar{a} \leq \pi$ is illustrated in Figure 9, which shows plots of the semi-width $a$, the contact angle $\beta$, and the maximum thickness $h_{\mathrm{m}}$ as functions of the scaled angle $\alpha / \pi$ for a range of values of $\bar{\beta}$ when $\bar{Q}=1$ and $\bar{a}=2(<\pi)$. In particular, Figure 9 shows that in this case pinning and de-pinning occur for $\bar{\beta}>\bar{\beta}_{\mathrm{c}} \simeq 1.0249$.

\section{$5.2 \quad \bar{a}>\pi$}

When $\bar{a}>\pi$, as in the case $a_{\min }<\bar{a} \leq \pi$, the rivulet runs from $\alpha=0$ with constant non-zero contact angle $\beta=\bar{\beta}>0$ but decreasing semi-width $a$ as described in section 3 until it reaches the value $a=\bar{a}$ at $\alpha=\alpha_{\text {pin }}\left(0<\alpha_{\text {pin }}<\alpha_{\mathrm{c}}\right)$ at which the contact lines pin. The rivulet then runs from $\alpha=\alpha_{\text {pin }}$ with constant semi-width $a=\bar{a}$ but decreasing contact angle $\beta$ as described in section 4 until, unlike in the case $a_{\min }<\bar{a} \leq \pi$, it reaches the critical azimuthal angle $\alpha=\alpha_{\mathrm{c}}\left(\pi / 2<\alpha_{\mathrm{c}}<\pi\right)$ at which the contact angle $\beta$ reaches the value zero and the contact lines de-pin. The rivulet then runs from $\alpha=\alpha_{\mathrm{c}}$ to $\alpha=\pi$ with zero contact angle $\beta=0$, decreasing semi-width $a=\pi / m$ and increasing maximum thickness $h_{\mathrm{m}}=h_{\mathrm{m} 0}$ as described in section 3 . In the special case of pinning at zero contact angle $\beta=\bar{\beta}=0$ pinning and de-pinning do not occur. In the limit $\bar{\beta} \rightarrow \infty$ we have $\alpha_{\text {pin }} \rightarrow 0^{+}$and so recover the solution for a rivulet with constant semi-width $a=\bar{a}>\pi$ described in section 4 .

The behaviour when $\bar{a}>\pi$ is illustrated in Figure 10, which shows plots of the semi-width $a$, the contact angle $\beta$, and the maximum thickness $h_{\mathrm{m}}$ as functions of the scaled angle $\alpha / \pi$ for a range of values of $\bar{\beta}$ when $\bar{Q}=1$ and $\bar{a}=5(>\pi)$. In particular, Figure 10 shows that pinning and de-pinning occur for all $\bar{\beta}>0$, and that in this case de-pinning occurs at the scaled critical azimuthal angle 

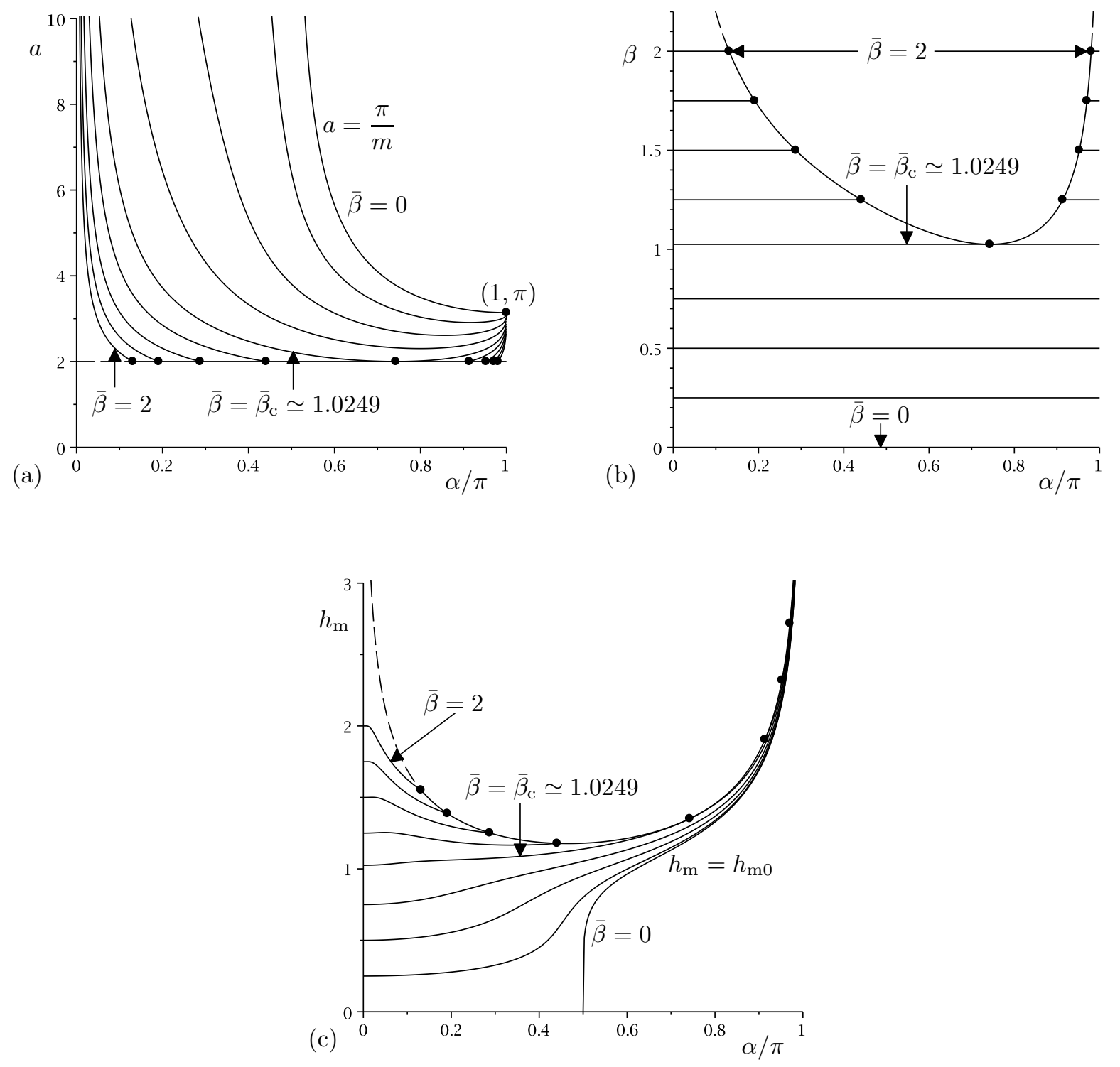

Figure 9: Plots of (a) the semi-width $a$, (b) the contact angle $\beta$, and (c) the maximum thickness $h_{\mathrm{m}}$ as functions of the scaled angle $\alpha / \pi$ for $\bar{\beta}=0,0.25,0.5,0.75, \bar{\beta}_{\mathrm{c}} \simeq 1.0249,1.25,1.5,1.75,2$ when $\bar{Q}=1$ for a rivulet whose contact lines pin at $a=\bar{a}=2(<\pi)$. The corresponding solutions for a rivulet with constant semi-width $\bar{a}=2$ are shown with dashed lines. Pinning and de-pinning occur for $\bar{\beta}>\bar{\beta}_{\mathrm{c}} \simeq 1.0249$, and the points at which this happens are denoted by dots. 
$\alpha_{\mathrm{c}} / \pi \simeq 0.6292$ for all $\bar{\beta}>0$.

\subsection{Rivulet Profiles}

Figure 11 shows typical cross-sectional profiles of the rivulet in the cases (a) $\bar{a}=2(<\pi)$ and $\bar{\beta}=0.5\left(<\bar{\beta}_{\mathrm{c}} \simeq 1.0249\right)$, (b) $\bar{a}=2(<\pi)$ and $\bar{\beta}=1.5\left(>\bar{\beta}_{\mathrm{c}}\right)$, and (c) $\bar{a}=5(>\pi)$ and $\bar{\beta}=1$, and, in order to clarify what might appear to be a rather complicated situation, Figure 12 shows sketches of the rivulet in the same three cases, namely (a) $\bar{a}<\pi$ and $0<\bar{\beta}<\bar{\beta}_{\mathrm{c}}$, in which the rivulet is never pinned, (b) $\bar{a}<\pi$ and $\bar{\beta}>\bar{\beta}_{\mathrm{c}}$, in which the rivulet is pinned in the interval $\alpha_{\text {pin }}<\alpha<\alpha_{\text {depin }}$, and (c) $\bar{a}>\pi$, in which the rivulet is pinned in the interval $\alpha_{\text {pin }}<\alpha<\alpha_{\mathrm{c}}$ and has zero contact angle in the interval $\alpha_{\mathrm{c}} \leq \alpha \leq \pi$.

\section{De-Pinning and Re-Pinning of a Rivulet with Constant Width $a=\bar{a}$ at $\beta=\bar{\beta}$}

In section 5 we described the pinning and de-pinning of a rivulet with constant contact angle $\beta=\bar{\beta}$ at $a=\bar{a}$. In this section we describe the corresponding situation involving the de-pinning and re-pinning of a rivulet with constant width $a=\bar{a}$ at $\beta=\bar{\beta}>0$. As we described in section 4 , for a narrow rivulet with $\bar{a}<\pi$ the contact angle $\beta$ of a slowly varying rivulet with constant semi-width $a=\bar{a}$ is unbounded at $\alpha=0$ and $\alpha=\pi$, and has a single minimum value of $\beta=\beta_{\min }$ at $\alpha=\alpha_{\min }$ on the lower half of the cylinder, while for a wide rivulet with $\bar{a}>\pi$ the contact angle is unbounded at $\alpha=0$ and decreases to the value zero at $\alpha=\alpha_{\mathrm{c}}$. In section 4 we showed how there can be steady flow of a wide rivulet all the way from $\alpha=0$ to $\alpha=\pi$ when the contact lines de-pin when the contact angle reaches its minimum physically realisable value of zero, i.e. at $\alpha=\alpha_{\mathrm{c}}$. More generally, however, the contact lines could de-pin at a non-zero value of the contact angle, denoted by $\beta=\bar{\beta}>0$. Evidently, as in section 5 , the behaviour of the rivulet in this situation will be qualitatively different for $\bar{a} \leq \pi$ and $\bar{a}>\pi$.

\section{$6.1 \quad \bar{a} \leq \pi$}

When $\bar{a}<\pi$ and $\bar{\beta}<\beta_{\min }$ the contact angle is always greater than or equal to $\bar{\beta}$ and hence depinning and re-pinning do not occur, and so the rivulet behaves exactly as described in section 4 . However, when $\bar{a}<\pi$ and $\bar{\beta} \geq \beta_{\text {min }}$ the rivulet runs from $\alpha=0$ with constant semi-width $a=\bar{a}$ but decreasing contact angle $\beta$ as described in section 4 until it reaches the value $\beta=\bar{\beta}$ at $\alpha=\alpha_{\text {depin }}$ 

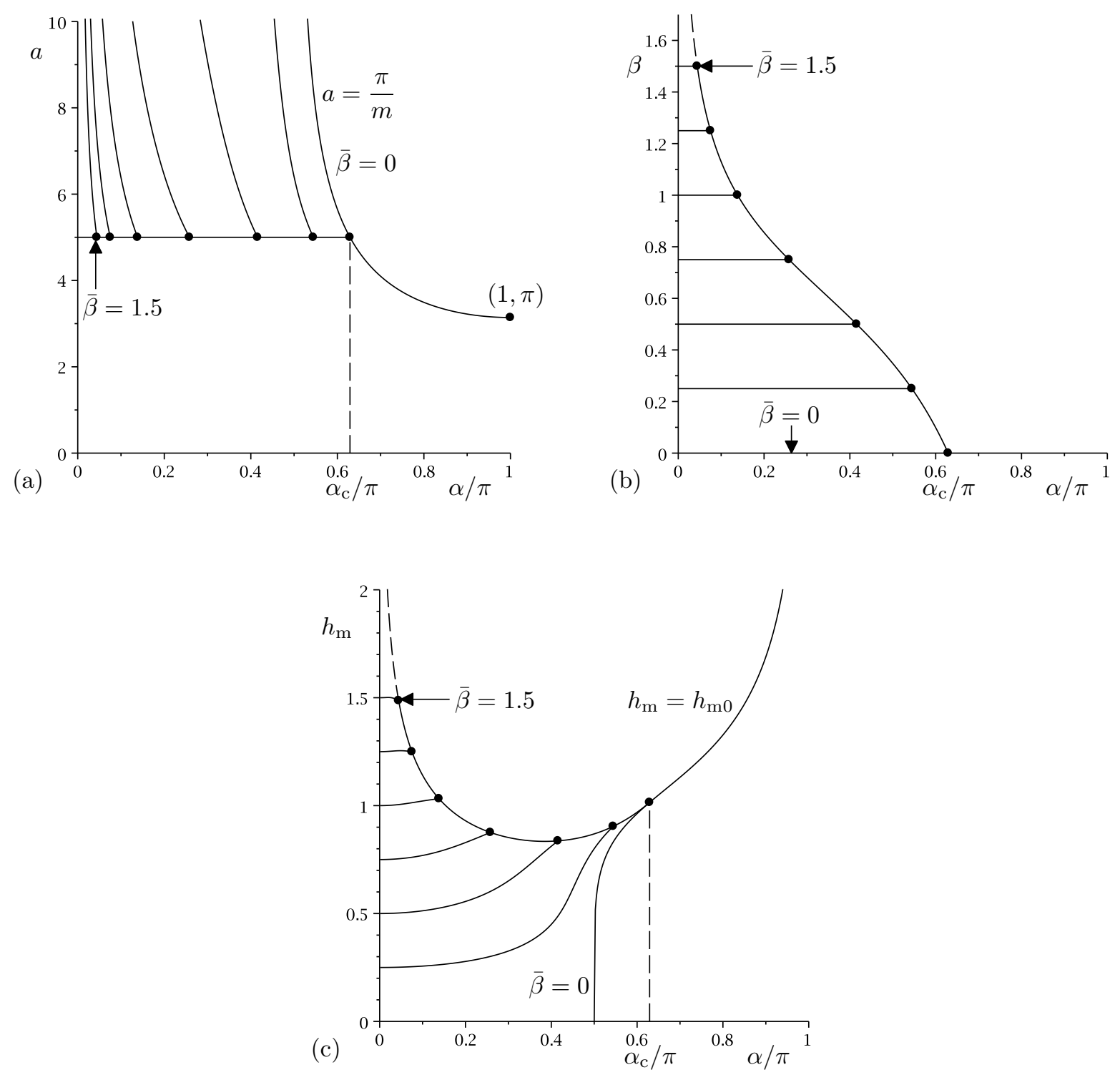

Figure 10: Plots of (a) the semi-width $a$, (b) the contact angle $\beta$, and (c) the maximum thickness $h_{\mathrm{m}}$ as functions of the scaled angle $\alpha / \pi$ for $\bar{\beta}=0,0.25, \ldots, 1.5$ when $\bar{Q}=1$ for a rivulet whose contact lines pin at $a=\bar{a}=5(>\pi)$. The corresponding solutions for a rivulet with constant semi-width $\bar{a}=5$ are shown with dashed lines. Pinning and de-pinning occur for all $\bar{\beta}>0$, and the points at which this happens are denoted by dots. The vertical dashed lines show the scaled critical azimuthal angle $\alpha_{\mathrm{c}} / \pi \simeq 0.6292$ at which de-pinning occurs for all $\bar{\beta}>0$. 

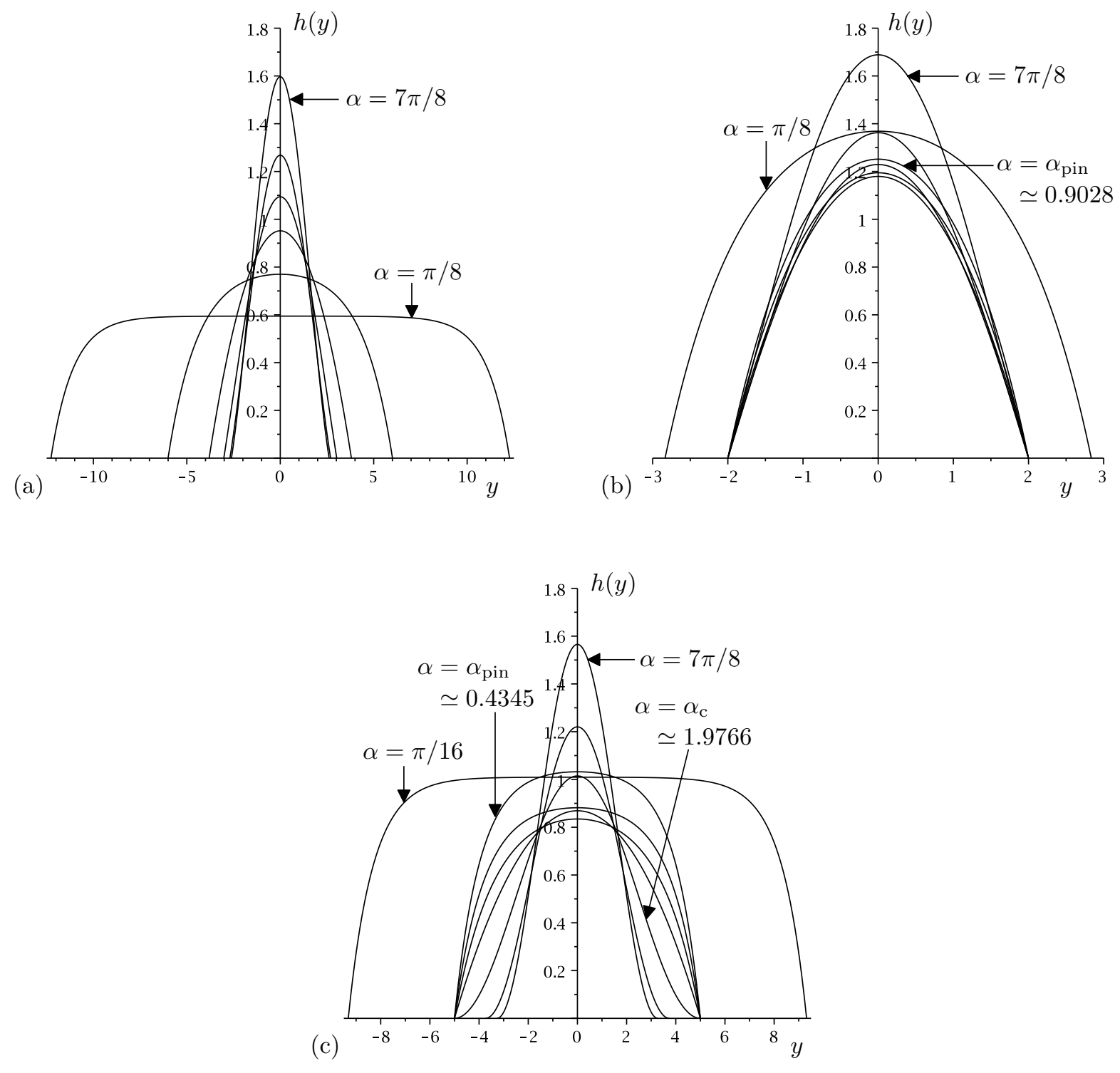

Figure 11: Cross-sectional profiles $h(y)$ when $\bar{Q}=1$ in the cases (a) $\bar{a}=2(<\pi)$ and $\bar{\beta}=0.5(<$ $\left.\bar{\beta}_{\mathrm{c}} \simeq 1.0249\right)$ for $\alpha=\pi / 8, \pi / 4,3 \pi / 8, \pi / 2,5 \pi / 8,3 \pi / 4,7 \pi / 8,(\mathrm{~b}) \bar{a}=2(<\pi)$ and $\bar{\beta}=1.5\left(>\bar{\beta}_{\mathrm{c}}\right)$ for $\alpha=\pi / 8, \alpha_{\text {pin }} \simeq 0.9028,3 \pi / 8, \pi / 2,5 \pi / 8,3 \pi / 4,7 \pi / 8$, and (c) $\bar{a}=5(>\pi)$ and $\bar{\beta}=1$ for $\alpha=\pi / 16$, $\alpha_{\text {pin }} \simeq 0.4345, \pi / 4,3 \pi / 8, \pi / 2, \alpha_{\mathrm{c}} \simeq 1.9766,3 \pi / 4,7 \pi / 8$. For clarity, in part (b) no profiles are shown in the interval $\alpha_{\text {depin }} \simeq 2.9923 \leq \alpha \leq \pi$, and the three parts of this figure use the same vertical but different horizontal ranges. 

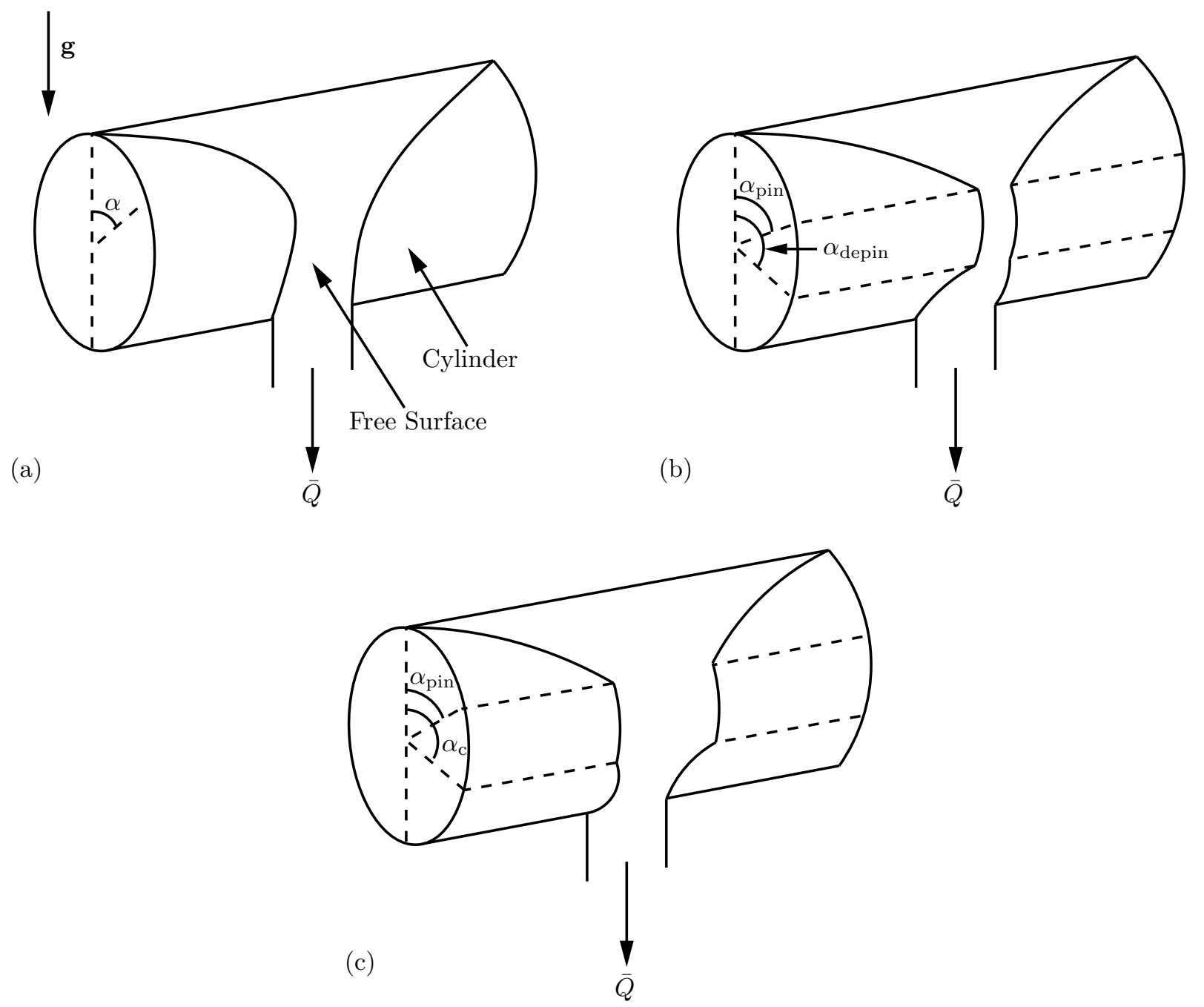

Figure 12: Sketches of a slowly varying rivulet with prescribed flux $\bar{Q}$ with (when not pinned with constant semi-width $a=\bar{a}$ and slowly varying contact angle $\beta$ ) constant non-zero contact angle $\beta=\bar{\beta}>0$ and slowly varying semi-width $a$ that runs from the top $\alpha=0$ to the bottom $\alpha=\pi$ of a large horizontal cylinder, in the cases (a) $\bar{a}<\pi$ and $0<\bar{\beta}<\bar{\beta}_{\mathrm{c}}$, in which the rivulet is never pinned, (b) $\bar{a}<\pi$ and $\bar{\beta}>\bar{\beta}_{\mathrm{c}}$, in which the rivulet is pinned in the interval $\alpha_{\text {pin }}<\alpha<\alpha_{\text {depin }}$, and (c) $\bar{a}>\pi$, in which the rivulet is pinned in the interval $\alpha_{\text {pin }}<\alpha<\alpha_{\mathrm{c}}$ and has zero contact angle in the interval $\alpha_{\mathrm{c}} \leq \alpha \leq \pi$. 
$\left(0<\alpha_{\text {depin }}<\alpha_{\min }\right)$ at which the contact lines de-pin. The rivulet then runs from $\alpha=\alpha_{\text {depin }}$ with constant contact angle $\beta=\bar{\beta}$ but varying semi-width $a$ as described in section 3 until it reaches $\alpha=\alpha_{\text {repin }}\left(\alpha_{\min }<\alpha_{\text {repin }}<\pi\right)$ at which the contact lines re-pin. The rivulet then runs from $\alpha=\alpha_{\text {repin }}$ to $\alpha=\pi$ with constant semi-width $a=\bar{a}$ but increasing contact angle $\beta$ as described in section 4. Here $\alpha=\alpha_{\text {depin }}$ and $\alpha=\alpha_{\text {repin }}$ are the appropriate solutions of the equation $Q=\bar{Q}$ with $Q$ given by (3), $a=\bar{a}$ and $\beta=\bar{\beta}$. In the marginal case $\bar{a}=\pi$ we have $\alpha_{\text {repin }}=\pi$ and so re-pinning does not occur. Expressed another way, de-pinning and re-pinning occur when $\bar{a}>\bar{a}_{\mathrm{c}}$, where the value of $\bar{a}_{\mathrm{c}}$ corresponds to $\beta_{\text {min }}=\bar{\beta}$. In the limit $\bar{a} \rightarrow \infty$ we have $\alpha_{\text {depin }} \rightarrow 0^{+}$and $\alpha_{\text {repin }} \rightarrow \pi^{-}$and so recover the solution for a rivulet with constant non-zero contact angle $\beta=\bar{\beta}>0$ described in section 3 .

\section{$6.2 \bar{a}>\pi$}

When $\bar{a}>\pi$, as in the case $\bar{a} \leq \pi$, the rivulet runs from $\alpha=0$ with constant semi-width $a=\bar{a}$ but decreasing contact angle $\beta$ as described in section 4 until it reaches the value $\beta=\bar{\beta}>0$ at $\alpha=\alpha_{\text {depin }}$ $\left(0<\alpha_{\text {depin }}<\alpha_{\mathrm{c}}\right)$ at which the contact lines de-pin. The rivulet then runs from $\alpha=\alpha_{\text {depin }}$ with contact angle $\beta=\bar{\beta}>0$ but varying semi-width $a$ as described in section 3 until, unlike in the case $\bar{a} \leq \pi$, it reaches $\alpha=\pi$. In the limit $\bar{a} \rightarrow \infty$ we have $\alpha_{\text {depin }} \rightarrow 0^{+}$and so again recover the solution for a rivulet with constant non-zero contact angle $\beta=\bar{\beta}>0$ described in section 3 .

\subsection{Rivulet Profiles}

The behaviour for $\bar{\beta}>0$ for both $\bar{a} \leq \pi$ and $\bar{a}>\pi$ is illustrated in Figure 13, which shows plots of the contact angle $\beta$, the scaled semi-width $a / \pi$, and the maximum thickness $h_{\mathrm{m}}$ as functions of the scaled angle $\alpha / \pi$ for a range of values of $\bar{a} / \pi$ when $\bar{Q}=1$ and $\bar{\beta}=1$. In particular, Figure 13 shows that in this case de-pinning and re-pinning occur for $\bar{a} / \pi>\bar{a}_{\mathrm{c}} / \pi \simeq 0.6446$ and de-pinning but no re-pinning occurs for $\bar{a} / \pi>1$. Figure 14 shows typical cross-sectional profiles of the rivulet in the cases (a) $\bar{\beta}=1$ and $\bar{a}=2\left(<\bar{a}_{\mathrm{c}} \simeq 2.0252\right)$ (b) $\bar{\beta}=1$ and $\bar{a}=2.5\left(\bar{a}_{\mathrm{c}}<\bar{a}<\pi\right)$, and (c) $\bar{\beta}=1$ and $\bar{a}=5(>\pi)$, and, in order to clarify what might again appear to be a rather complicated situation, Figure 15 shows sketches of the rivulet in the same three cases, namely (a) $\bar{a}<\bar{a}_{\mathrm{c}}<\pi$, in which the rivulet is never de-pinned, (b) $\bar{a}_{\mathrm{c}}<\bar{a}<\pi$, in which the rivulet is de-pinned in the interval $\alpha_{\text {depin }}<\alpha<\alpha_{\text {repin }}$, and (c) $\bar{a}>\pi$, in which the rivulet is de-pinned in the interval $\alpha_{\text {depin }}<\alpha \leq \pi$. 

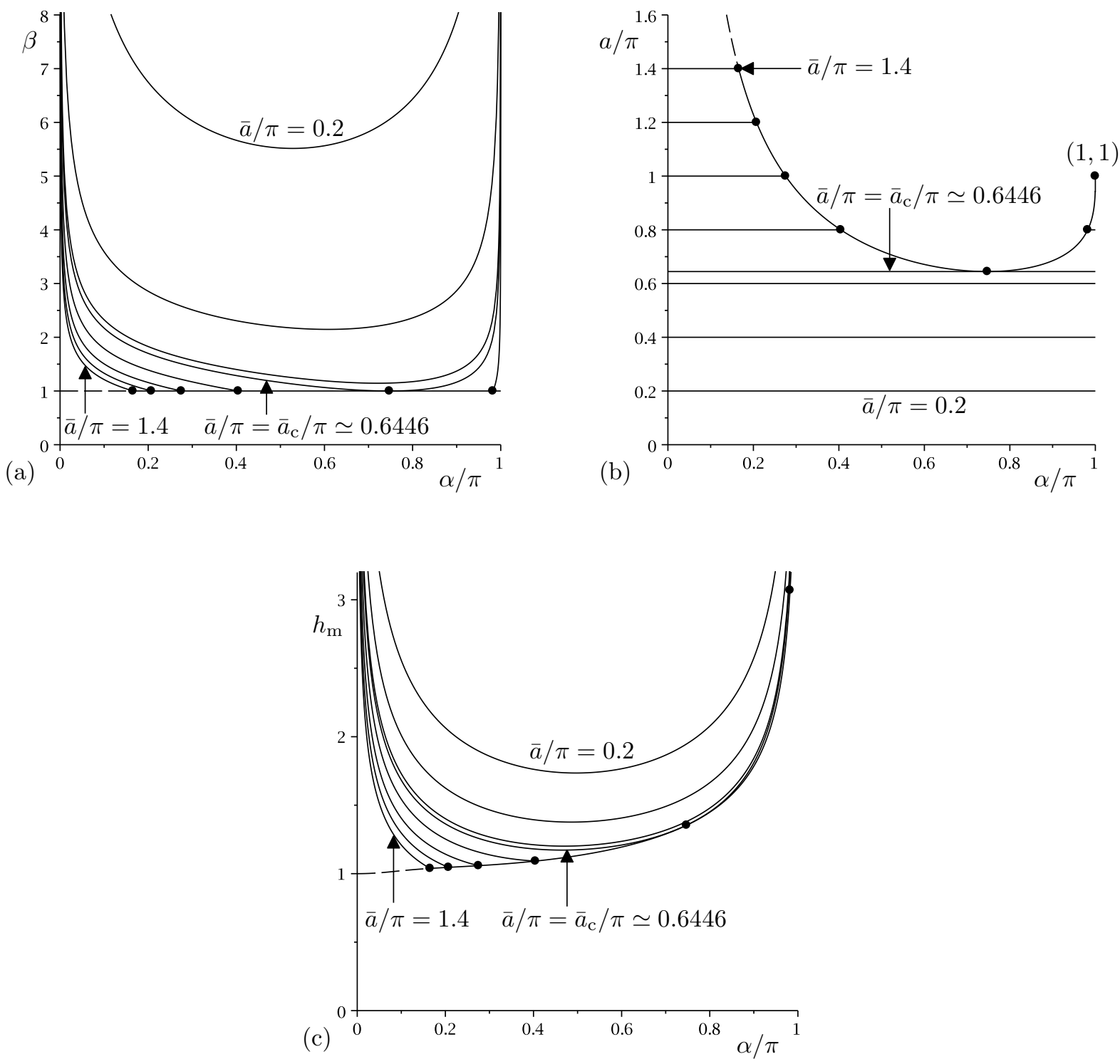

Figure 13: Plots of (a) the contact angle $\beta$, (b) the scaled semi-width $\bar{a} / \pi$, and (c) the maximum thickness $h_{\mathrm{m}}$ as functions of the scaled angle $\alpha / \pi$ for $\bar{a} / \pi=0.2,0.4,0.6, \bar{a}_{\mathrm{c}} / \pi \simeq 0.6446,0.8,1,1.2$, 1.4 when $\bar{Q}=1$ for a rivulet whose contact lines de-pin at non-zero contact angle $\beta=\bar{\beta}=1$. The corresponding solutions for a rivulet with constant contact angle $\beta=\bar{\beta}=1$ are shown with dashed lines. De-pinning and re-pinning occur for $\bar{a} / \pi>\bar{a}_{\mathrm{c}} / \pi \simeq 0.6446$, de-pinning but no re-pinning occurs for $\bar{a} / \pi>1$, and the points at which this happens are denoted by dots. 

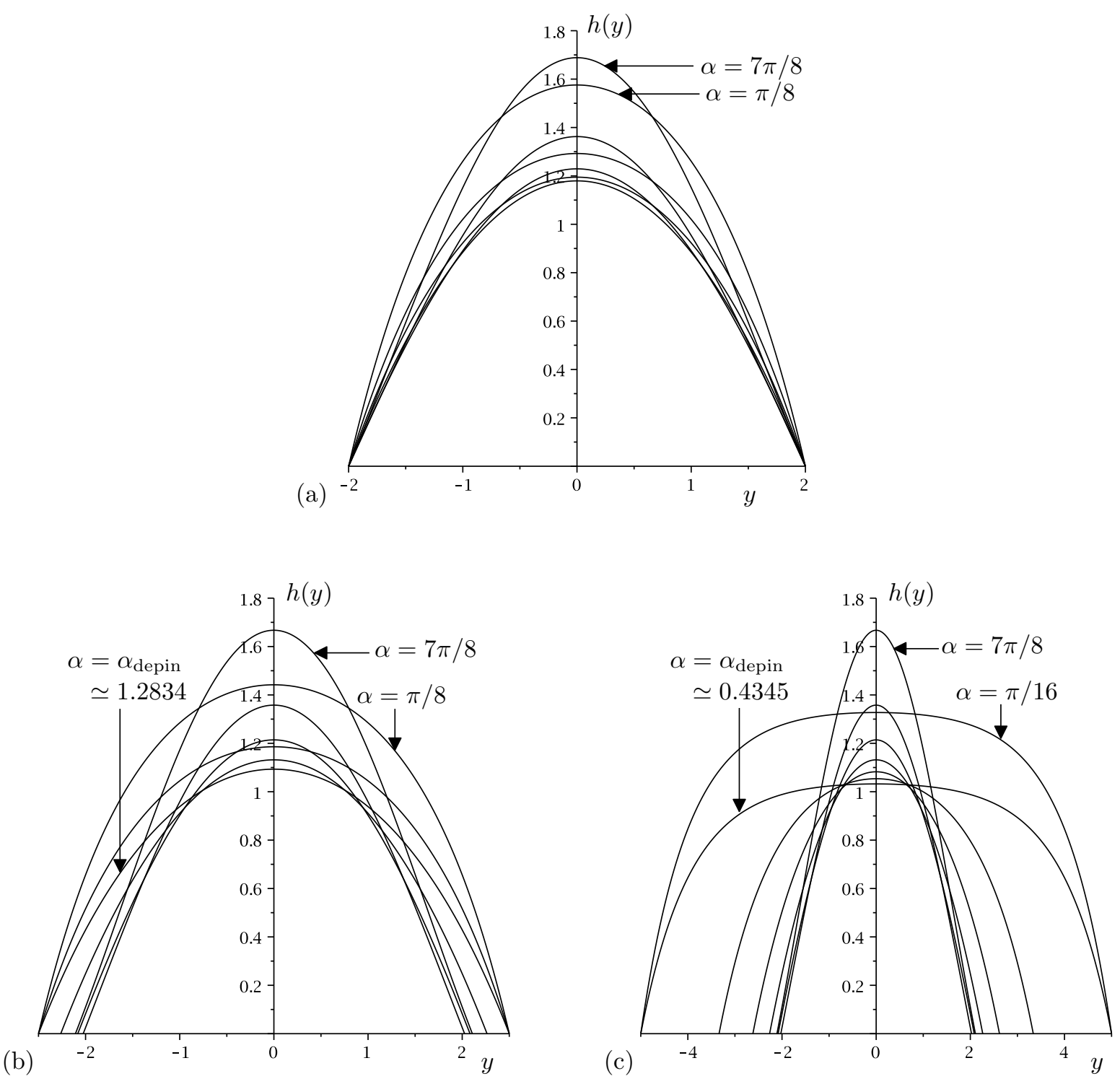

Figure 14: Cross-sectional profiles $h(y)$ when $\bar{Q}=1$ in the cases (a) $\bar{\beta}=1$ and $\bar{a}=2\left(<\bar{a}_{\mathrm{c}} \simeq 2.0252\right)$ for $\alpha=\pi / 8, \pi / 4,3 \pi / 8, \pi / 2,5 \pi / 8,3 \pi / 4,7 \pi / 8$, (b) $\bar{\beta}=1$ and $\bar{a}=2.5\left(\bar{a}_{\mathrm{c}}<\bar{a}<\pi\right)$ for $\alpha=\pi / 8$, $\pi / 4, \alpha_{\text {depin }} \simeq 1.2834,3 \pi / 8, \pi / 2,5 \pi / 8,3 \pi / 4,7 \pi / 8$, and $(\mathrm{c}) \bar{\beta}=1$ and $\bar{a}=5(>\pi)$ for $\alpha=\pi / 16$, $\alpha_{\text {depin }} \simeq 0.4345, \pi / 4,3 \pi / 8, \pi / 2,5 \pi / 8,3 \pi / 4,7 \pi / 8$. For clarity, in part (b) no profiles are shown in the interval $\alpha_{\text {repin }} \simeq 3.0814 \leq \alpha \leq \pi$, and the three parts of this figure use the same vertical but different horizontal ranges. 

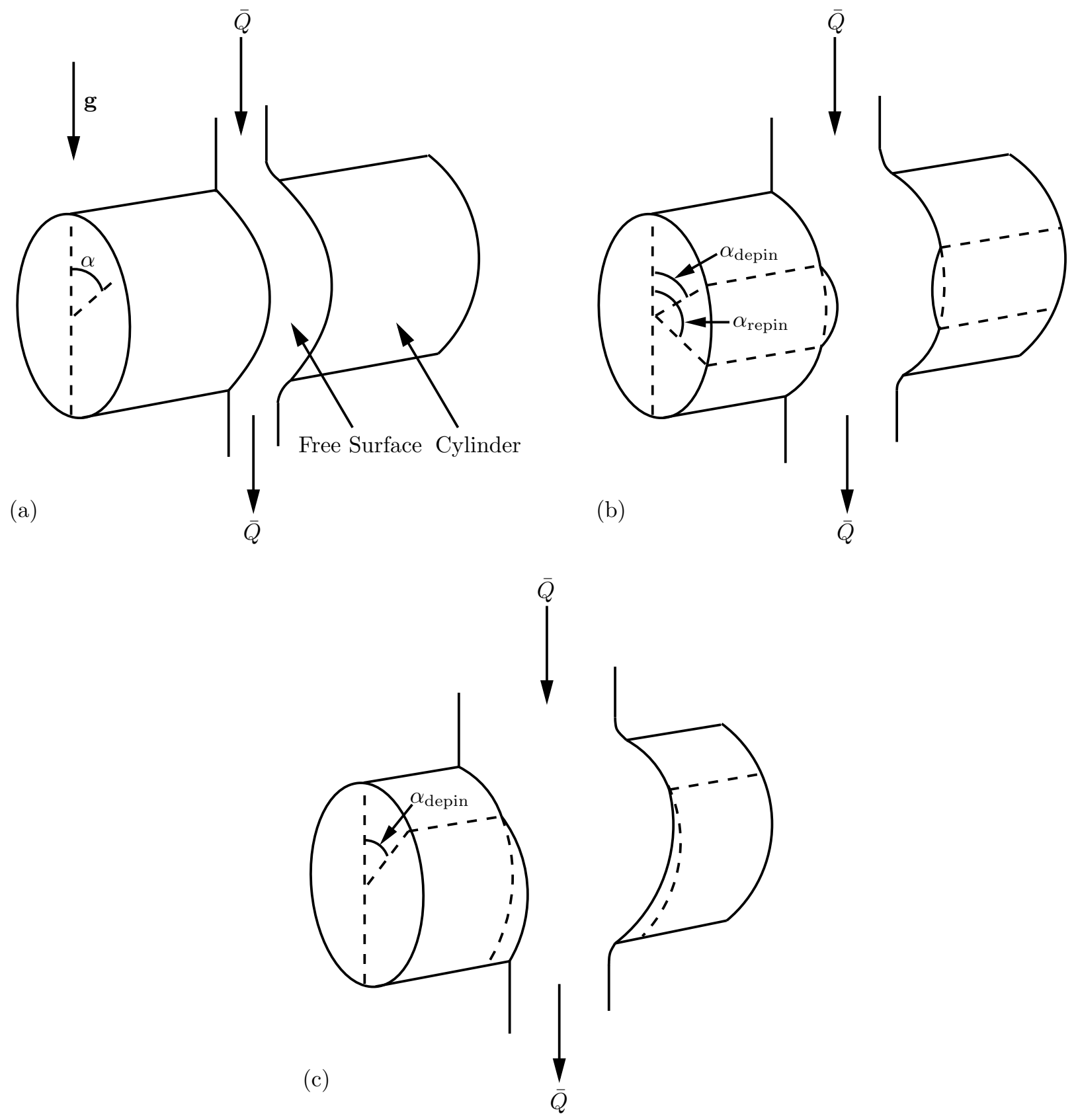

Figure 15: Sketches of a slowly varying rivulet with prescribed flux $\bar{Q}$ with (when not de-pinned with non-zero constant contact angle $\beta=\bar{\beta}>0$ and slowly varying semi-width $a$ ) constant semi-width $a=\bar{a}$ and slowly varying contact angle $\beta$ that runs from the top $\alpha=0$ to the bottom $\alpha=\pi$ of a large horizontal cylinder, in the cases (a) $\bar{a}<\bar{a}_{\mathrm{c}}<\pi$, in which the rivulet is never de-pinned, (b) $\bar{a}_{\mathrm{c}}<\bar{a}<\pi$, in which the rivulet is de-pinned in the interval $\alpha_{\text {depin }}<\alpha<\alpha_{\text {repin }}$, and (c) $\bar{a}>\pi$, in which the rivulet is de-pinned in the interval $\alpha_{\text {depin }}<\alpha \leq \pi$. 


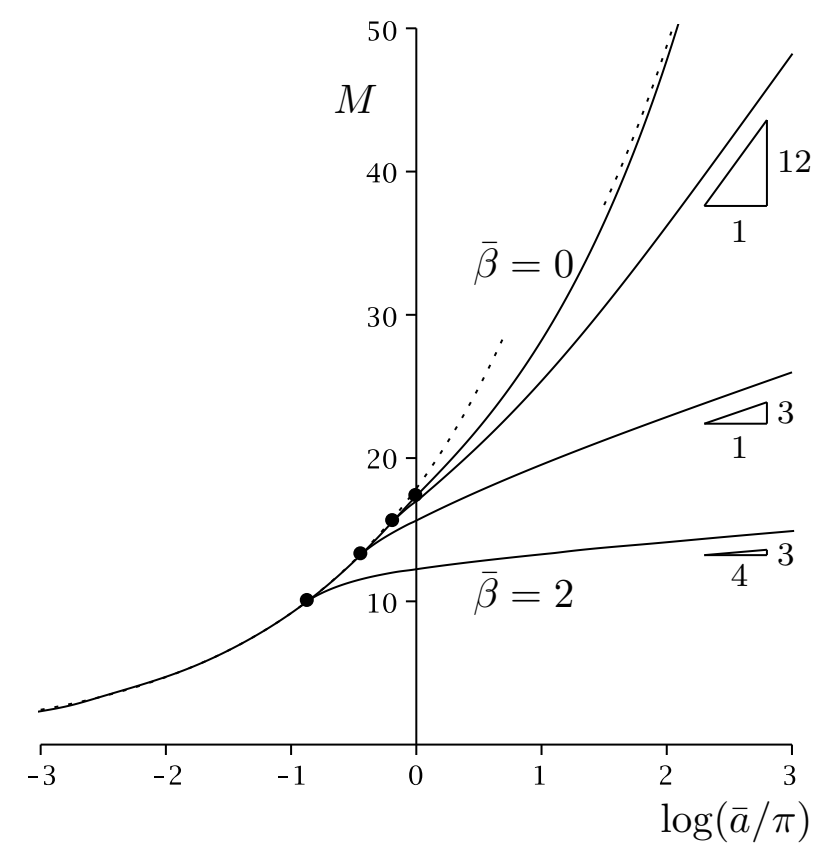

Figure 16: The mass of fluid on the cylinder $M$ for a rivulet whose contact lines de-pin at contact angle $\beta=\bar{\beta}$ plotted as a function of the logarithm of the scaled semi-width $\log (\bar{a} / \pi)$ for $\bar{\beta}=0,1 / 2$, 1, 2 when $\bar{Q}=1$, together with its leading order asymptotic behavour in the limits $\bar{a} \rightarrow 0^{+}$given by (27) and $\bar{a} \rightarrow \infty$ when $\bar{\beta}=0$ given by (28), shown with dotted lines. The triangles indicate the slopes $3 \bar{Q} / \bar{\beta}^{2}=12,3,3 / 4$, confirming the leading order asymptotic behaviour in the limit $\bar{a} \rightarrow \infty$ when $\bar{\beta}>0$ given by (29). De-pinning and re-pinning occur for $\bar{a} / \pi>\bar{a}_{\mathrm{c}} / \pi$, de-pinning but no re-pinning occurs for $\bar{a} / \pi>1$, and the points at which de-pinning first occurs are denoted by dots. 


\subsection{Mass of Fluid on the Cylinder}

The mass of fluid on the cylinder $M$ is given by

$$
M=\int_{0}^{\pi} \int_{-a}^{+a} h \mathrm{~d} y \mathrm{~d} \alpha=\int_{0}^{\frac{\pi}{2}} \frac{2 \beta(m a \operatorname{coth} m a-1)}{m^{2}} \mathrm{~d} \alpha+\int_{\frac{\pi}{2}}^{\pi} \frac{2 \beta(1-m a \cot m a)}{m^{2}} \mathrm{~d} \alpha .
$$

Figure 16 shows $M$ plotted as a function of the logarithm of the scaled semi-width $\log (\bar{a} / \pi)$ for a range of values of $\bar{\beta}$, and shows that $M$ is a monotonically increasing function of $\bar{a}$. Figure 16 also shows that in the limit of a narrow rivulet, $\bar{a} \rightarrow 0^{+}, M \rightarrow 0^{+}$according to

$$
M \sim\left(\frac{70 \bar{a}^{2} \bar{Q}}{9}\right)^{\frac{1}{3}} C \rightarrow 0^{+},
$$

while in the limit of a wide rivulet on the upper half of the cylinder, $\bar{a} \rightarrow \infty, M \rightarrow \infty$ according to

$$
M \sim\left(\frac{3 \bar{a}^{2} \bar{Q}}{2}\right)^{\frac{1}{3}} C \rightarrow \infty
$$

when $\bar{\beta}=0$ and

$$
M \sim \frac{3 \bar{Q}}{\bar{\beta}^{2}} \log \bar{a} \rightarrow \infty
$$

when $\bar{\beta}>0$, where the constant $C$ is again given by (10).

\section{Conclusions}

In the present work we showed how the solutions for the unidirectional flow of a thin rivulet with prescribed volume flux down an inclined planar substrate can be used to describe the locally unidirectional flow of a rivulet with constant width (i.e. pinned contact lines) but slowly varying contact angle as well as the possible pinning and subsequent de-pinning of a rivulet with constant contact angle and the possible de-pinning and subsequent re-pinning of a rivulet with constant width as they flow in the azimuthal direction from the top $\alpha=0$ to the bottom $\alpha=\pi$ of a large horizontal cylinder. We found that, despite being the same locally, the global behaviour of a rivulet with constant width can be very different from that of a rivulet with constant contact angle described by Duffy and Moffatt [20] and Duffy and Wilson [21]. Specifically, while a rivulet with constant non-zero contact angle $\beta=\bar{\beta}>0$ can always run from the top to the bottom of the cylinder, the behaviour of a rivulet with constant width $\bar{a}$ depends on the value of $\bar{a}$. In particular, while a narrow rivulet with constant semi-width $a=\bar{a} \leq \pi$ can run all the way from the top to the bottom of the cylinder, a wide rivulet with constant semi-width $a=\bar{a}>\pi$ can run from the top of the cylinder only to a critical azimuthal angle $\alpha=\alpha_{\mathrm{c}}$ given by (19). In section 4 we discussed the scenario in which the 
hitherto pinned contact lines of the rivulet de-pin at $\alpha=\alpha_{\mathrm{c}}$ and the rivulet runs from $\alpha=\alpha_{\mathrm{c}}$ to the bottom of the cylinder with zero contact angle but slowly varying semi-width $a=\pi / m$, as sketched in Figure 8.

In section 5 we described the pinning and de-pinning of a rivulet with constant contact angle $\beta=\bar{\beta}$ at $a=\bar{a}$. In particular, we showed that when $\bar{a} \leq \pi$ the rivulet is pinned in the interval

$\alpha_{\text {pin }}<\alpha<\alpha_{\text {depin }}$ for $\bar{\beta}>\bar{\beta}_{\mathrm{c}}$, but that when $\bar{a}>\pi$ the rivulet is pinned in the interval $\alpha_{\text {pin }}<\alpha<\alpha_{\mathrm{c}}$ and has zero contact angle in the interval $\alpha_{\mathrm{c}} \leq \alpha \leq \pi$ for all $\bar{\beta}>0$, as sketched in Figure 12 . In section 6 we described the corresponding situation involving the de-pinning and re-pinning of a rivulet with constant semi-width $a=\bar{a}$ at a non-zero contact angle $\beta=\bar{\beta}>0$ which generalises the de-pinning at zero contact angle discussed in section 4. In particular, we showed that when $\bar{a} \leq \pi$ the rivulet is de-pinned in the interval $\alpha_{\text {depin }}<\alpha<\alpha_{\text {repin }}$ for $\bar{a}>\bar{a}_{\mathrm{c}}$, but that when $\bar{a}>\pi$ the rivulet is de-pinned in the interval $\alpha_{\text {depin }}<\alpha \leq \pi$, as sketched in Figure 15. In the latter situation, the mass of fluid on the cylinder was found to be a monotonically increasing function of the constant semi-width $\bar{a}$.

\section{Acknowledgements}

The first author (CP) gratefully acknowledges the financial support of the University of Strathclyde via a Postgraduate Research Scholarship. Part of this work was undertaken while the corresponding author (SKW) was a Visiting Fellow in the Department of Mechanical and Aerospace Engineering, School of Engineering and Applied Science, Princeton University, USA, and part of it was undertaken while he was a Visiting Fellow in the Oxford Centre for Collaborative Applied Mathematics (OCCAM), Mathematical Institute, University of Oxford, United Kingdom. This publication was based on work supported in part by Award No KUK-C1-013-04, made by King Abdullah University of Science and Technology (KAUST). A preliminary version of this work, discussing only the depinning of a rivulet with constant width at zero contact angle, appears as OCCAM preprint number $12 / 43[35]$.

\section{Authors' Short Vitae}

Colin Paterson is a postgraduate student in the Department of Mathematics and Statistics at the University of Strathclyde and is currently undertaking theoretical research into various situations in which a thin layer of fluid is subject to an external airflow. The present study arose directly from 
his ongoing research into rivulet flow in the presence of an external airflow.

Professor Stephen Wilson is Professor of Applied Mathematics at the University of Strathclyde and has research interests in the application of mathematical techniques to a wide range of realworld problems in fluid mechanics. He is Joint Editor-in-Chief (with Professor Tom Witelski, Duke University, USA) of the Journal of Engineering Mathematics and is a Fellow of the Institute for Mathematics and its Applications (IMA).

Dr Brian Duffy is a Reader in Mathematics at the University of Strathclyde and has a long standing interest in the mathematical analysis of a wide range of problems in fluid mechanics, notably thin-film flows and flows of non-Newtonian fluids (including liquid crystals). Together with Professor Wilson and Gavin Dunn (University of Strathclyde) and Dr Khellil Sefiane and Samuel David (University of Edinburgh) he won the 2009 Institute of Physics Printing and Graphics Science Group Prize for his work on "a fundamental study of droplet evaporation".

\section{References}

[1] P. Vlasogiannis, G. Karagiannis, P. Argyropoulos, and V. Bontozoglou, "Air-water two-phase flow and heat transfer in a plate heat exchanger," Int. J. Multiphase Flow 28, 757-772 (2002).

[2] R. Maiti, R. Khanna, and K. D. P. Nigam, "Hysteresis in trickle-bed reactors: a review," Ind. Eng. Chem. Res. 45, 5185-5198 (2006).

[3] S. F. Kistler and P. M. Schweizer (Eds), Liquid Film Coating, Chapman and Hall (1997).

[4] M. E. Labib, S. Dukhin, J. Murawski, Y. Tabani, and R. Lai, "Surfactant influence on rivulet droplet flow in minitubes and capillaries and its downstream evolution," Adv. Col. Int. Sci. 166, 60-86 (2011).

[5] G. D. Towell and L. B. Rothfeld, "Hydrodynamics of rivulet flow," A. I. Ch. E. J. 12, 972-980 (1966).

[6] R. F. Allen and C. M. Biggin, "Longitudinal flow of a lenticular liquid filament down an inclined plane," Phys. Fluids 17, 287-291 (1974).

[7] M. Bentwich, D. Glasser, J. Kern, and D. Williams, "Analysis of rectilinear rivulet flow," A. I. Ch. E. J. 22, 772-779 (1976).

[8] S. H. Davis, "Moving contact lines and rivulet instabilities. Part 1. The static rivulet," J. Fluid Mech. 98, 225-242 (1980).

[9] R. H. Weiland and S. H. Davis, "Moving contact lines and rivulet instabilities. Part 2. Long waves on flat rivulets," J. Fluid Mech. 107, 261-280 (1981). 
[10] G. W. Young and S. H. Davis, "Rivulet instabilities," J. Fluid Mech. 176, 1-31 (1987).

[11] P. Schmuki and M. Laso, "On the stability of rivulet flow," J. Fluid Mech. 215, 125-143 (1990).

[12] P. A. Kuibin, "An asymptotic description of the rivulet flow along an inclined cylinder," Russ. J. Eng. Thermophys. 6, 33-45 (1996).

[13] S. V. Alekseenko, P. I. Geshev, and P. A. Kuibin, "Free-boundary fluid flow on an inclined cylinder," Physics-Doklady 42, 269-272 (1997).

[14] S. V. Alekseenko, A. V. Bobylev, and D. M. Markovich, "Rivulet flow on the outer surface of an inclined cylinder," J. Eng. Thermophys. 17, 259-272 (2008).

[15] C. A. Perazzo and J. Gratton, "Navier-Stokes solutions for parallel flow in rivulets on an inclined plane," J. Fluid Mech. 507, 367-379 (2004).

[16] A. J. Tanasijczuk, C. A. Perazzo, and J. Gratton, "Navier-Stokes solutions for steady parallel-sided pendent rivulets," Euro. J. Mech. B 29, 465-471 (2010).

[17] T. G. Myers, H. X. Liang, and B. Wetton, "The stability and flow of a rivulet driven by interfacial shear and gravity," Int. J. Nonlinear Mech. 39, 1239-1249 (2004).

[18] S. K. Wilson and B. R. Duffy, "Unidirectional flow of a thin rivulet on a vertical substrate subject to a prescribed uniform shear stress at its free surface," Phys. Fluids 17, 108105 (2005).

[19] E. S. Benilov, "On the stability of shallow rivulets," J. Fluid Mech. 636, 455-474 (2009).

[20] B. R. Duffy and H. K. Moffatt, "Flow of a viscous trickle on a slowly varying incline," Chem. Eng. J. 60, 141-146 (1995).

[21] B. R. Duffy and S. K. Wilson, "A rivulet of perfectly wetting fluid with temperature-dependent viscosity draining down a uniformly heated or cooled slowly varying substrate," Phys. Fluids 15, 3236-3239 (2003).

[22] S. K. Wilson and B. R. Duffy, "On the gravity-driven draining of a rivulet of viscous fluid down a slowly varying substrate with variation transverse to the direction of flow," Phys. Fluids 10, 13-22 (1998).

[23] D. Holland, B. R. Duffy, and S. K. Wilson, "Thermocapillary effects on a thin viscous rivulet draining steadily down a uniformly heated or cooled slowly varying substrate," J. Fluid Mech. 441, 195-221 (2001).

[24] S. K. Wilson, B. R. Duffy, and A. B. Ross, "On the gravity-driven draining of a rivulet of viscoplastic material down a slowly varying substrate," Phys. Fluids 14, 555-571 (2002).

[25] S. K. Wilson and B. R. Duffy, "Strong temperature-dependent-viscosity effects on a rivulet draining down a uniformly heated or cooled slowly varying substrate," Phys. Fluids 15, 827-840 (2003). 
[26] S. K. Wilson and B. R. Duffy, "A rivulet of perfectly wetting fluid draining steadily down a slowly varying substrate," IMA J. Appl. Math. 70, 293-322 (2005).

[27] J. M. Sullivan, S. K. Wilson, and B. R. Duffy, "A thin rivulet of perfectly wetting fluid subject to a longitudinal surface shear stress," Q. J. Mech. Appl. Math. 61, 25-61 (2008).

[28] G. Ribatski and A. M. Jacobi, "Falling-film evaporation on horizontal tubes - a critical review," Int. J. Refrig. 28, 635-653 (2005).

[29] J. Mitrovic, "Flow structures of a liquid film falling on horizontal tubes," Chem. Eng. Technol. 28, 684-694 (2005).

[30] R. Hunt, "The numerical solution of parabolic free boundary problems arising from thin film flows," J. Comp. Phys. 84, 377-402 (1989).

[31] R. Hunt, "The numerical solution of the thin film flow surrounding a horizontal cylinder resulting from a vertical cylindrical jet," Int. J. Numer. Meth. Fluids 14, 539-556 (1992).

[32] H. K. Moffatt, "Behaviour of a viscous film on the outer surface of a rotating cylinder," J. Méc. 16, 651-673 (1977).

[33] G. A. Leslie, S. K. Wilson, and B. R. Duffy, "Three-dimensional coating and rimming flow: a ring of fluid on a rotating horizontal cylinder," J. Fluid Mech. 716, 51-82 (2013).

[34] J. Deans and S. Kucuka, "The formation of banded condensate films in weak ammonia-water mixtures," J. Heat Transfer 133, 101505 (2011).

[35] C. Paterson, S. K. Wilson, and B. R. Duffy, "Gravity-driven draining of a thin rivulet with constant width down a slowly varying substrate," Oxford Centre for Collaborative Applied Mathematics (OCCAM) Preprint Number 12/43 (2012). 